Revue des patrimoines

$14 \mid 2010$

Le cavalier du Pont-Neuf : histoire, restauration et secrets de la statue équestre de Henri IV

\title{
Restauration et conservation des « trésors » cachés dans le cavalier de bronze
}

Nelly Cauliez, Isabelle Blivet, Myriam Kriche et Laurent Martin-Saint-Clair

\section{(2) OpenEdition}

Journals

Édition électronique

URL : http://journals.openedition.org/insitu/7044

DOI : 10.4000/insitu.7044

ISSN : 1630-7305

Éditeur

Ministère de la culture

Référence électronique

Nelly Cauliez, Isabelle Blivet, Myriam Kriche et Laurent Martin-Saint-Clair, «Restauration et conservation des «trésors » cachés dans le cavalier de bronze », In Situ [En ligne], 14 | 2010, mis en ligne le 10 avril 2012, consulté le 03 mai 2019. URL : http://journals.openedition.org/insitu/7044 ; DOI : 10.4000/insitu.7044

Ce document a été généré automatiquement le 3 mai 2019.

\section{(†) $\ominus$

In Situ Revues des patrimoines est mis à disposition selon les termes de la licence Creative Commons Attribution - Pas d'Utilisation Commerciale - Pas de Modification 4.0 International. 


\section{Restauration et conservation des «trésors » cachés dans le cavalier de bronze}

Nelly Cauliez, Isabelle Blivet, Myriam Kriche et Laurent Martin-Saint-Clair

\section{La découverte}

1 La rénovation en novembre 2004 de la statue équestre de Henri IV située sur le Pont-Neuf à Paris a permis d'en retirer sept boîtes constituées de bois de cèdre, de buis et de plomb ou d'étain qui renfermaient divers documents.

2 On connaissait l'existence de quatre d'entre elles, logées dans le ventre du cheval et qui viennent compléter la série des pièces déjà conservée aux Archives nationales. En revanche, on ignorait que trois autres boîtes étaient cachées dans le bras et dans la tête du cavalier.

\section{L'état de conservation et le protocole d'intervention}

3 Près de deux siècles séparent la pose des boîtes dans la statue, le jour de l'inauguration en août 1818, des découvertes réalisées en 2004. Ce long séjour dans des conditions inadaptées n'a pas été sans conséquence pour la conservation des documents dont les matériaux constitutifs sont très hétérogènes : papier, parchemin, cuir, métal, bois...

Ces objets, pour la plupart très dégradés, ont subi de multiples facteurs de détérioration :

- des variations de température et d'humidité au sein de la structure de bronze (inférieures à $0^{\circ} \mathrm{C}$ en hiver et supérieures à $80^{\circ} \mathrm{C}$ en été),

- une pollution atmosphérique accrue (le Pont-Neuf étant une voie de circulation très empruntée),

- la présence ou le développement d'agents biologiques (insectes, champignons),

- une dégradation inhérente aux matériaux eux-mêmes (encre métallogallique). 
Les conservateurs, les restaurateurs et les scientifiques des Archives nationales ont analysé les documents partiellement déchiffrables. Ils ont décidé de restaurer et conserver les boîtes et leur contenu comme des objets archéologiques ${ }^{1}$ empreints d'une histoire peu commune, en limitant leurs interventions. L'ensemble des actions directement entreprises sur ces objets a eu pour objectif d'arrêter le processus actif de détérioration ou de les renforcer structurellement. Ces actions n'ont été mises en œuvre que parce que l'existence même des biens était menacée, à relativement court terme, par leur extrême fragilité ou la vitesse de leur détérioration².

6 La valeur historique de ces documents d'archives est indéniable, tant par leur matérialité que par leur contenu. En ce sens il était essentiel que les traitements soient le moins interventionnistes possible pour qu'ils n'interfèrent pas ou peu avec les informations dont ils étaient porteurs afin de préserver toutes les données archéologiques ${ }^{3}$. Par conséquent, il a été préféré que les traitements restent visibles pour autoriser une lecture directe des informations matérielles et le protocole suivant a été appliqué :

- analyse et traitement de désinfection car l'état sanitaire de l'ensemble était préoccupant,

- pour les boîtes : traitement du plomb par nettoyage à sec et à l'acide chlorhydrique très dilué et traitement du bois par infiltration de résines acryliques stables, atténuation de la peste de l'étain par nettoyage aqueux ${ }^{4}$,

- pour les objets : nettoyage à sec (papier, parchemin...), consolidations des parties fragiles, mise à plat des documents qui ont subi d'importantes variations dimensionnelles.

7 Seules les boîtes découvertes dans la tête et le bras du cavalier de bronze ont fait l'objet d'une analyse particulière.

\section{Analyses complémentaires}


Figure 1

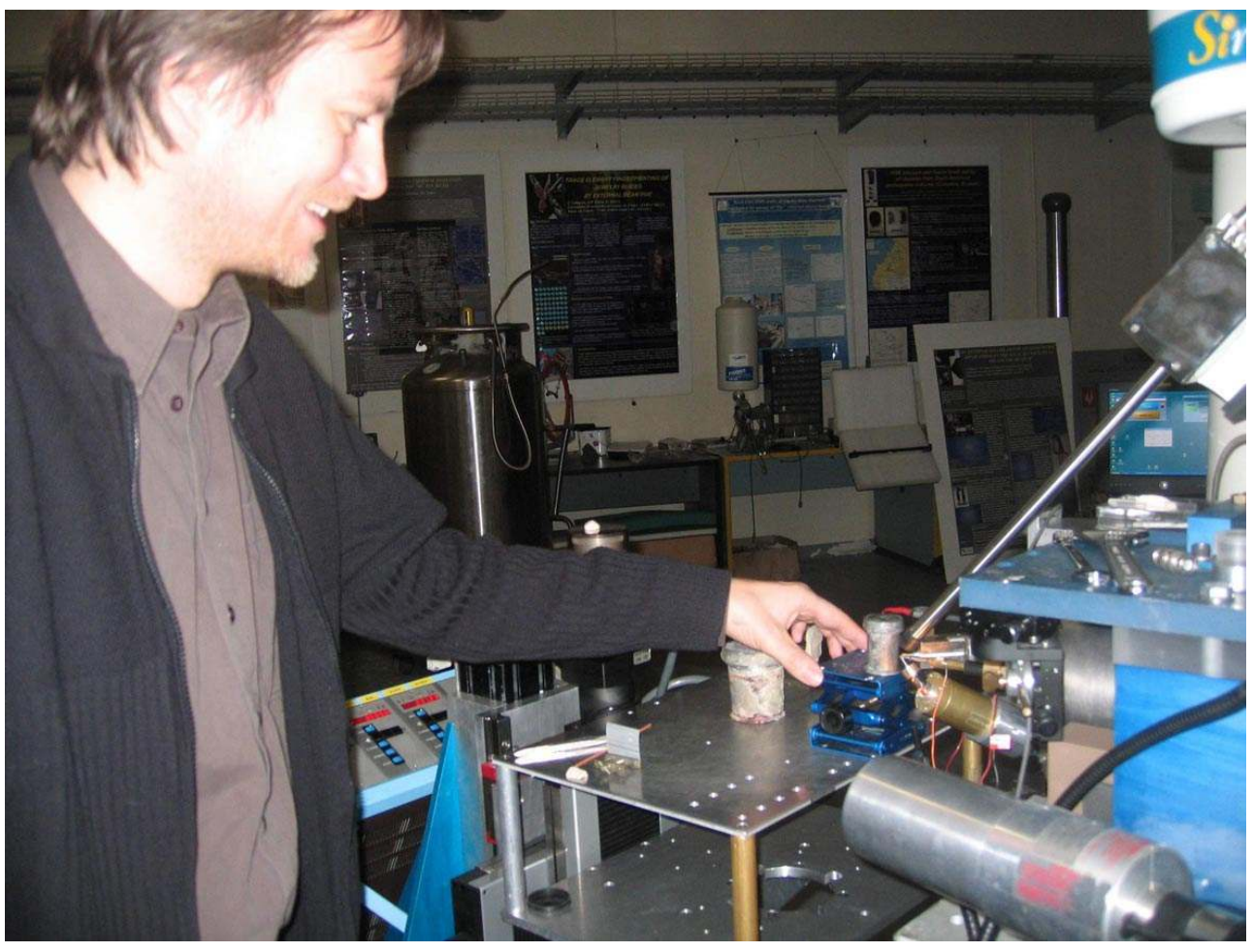

Tomographie d'une des boîtes en étain

(c) C2RMF, 2006 
Figure 2

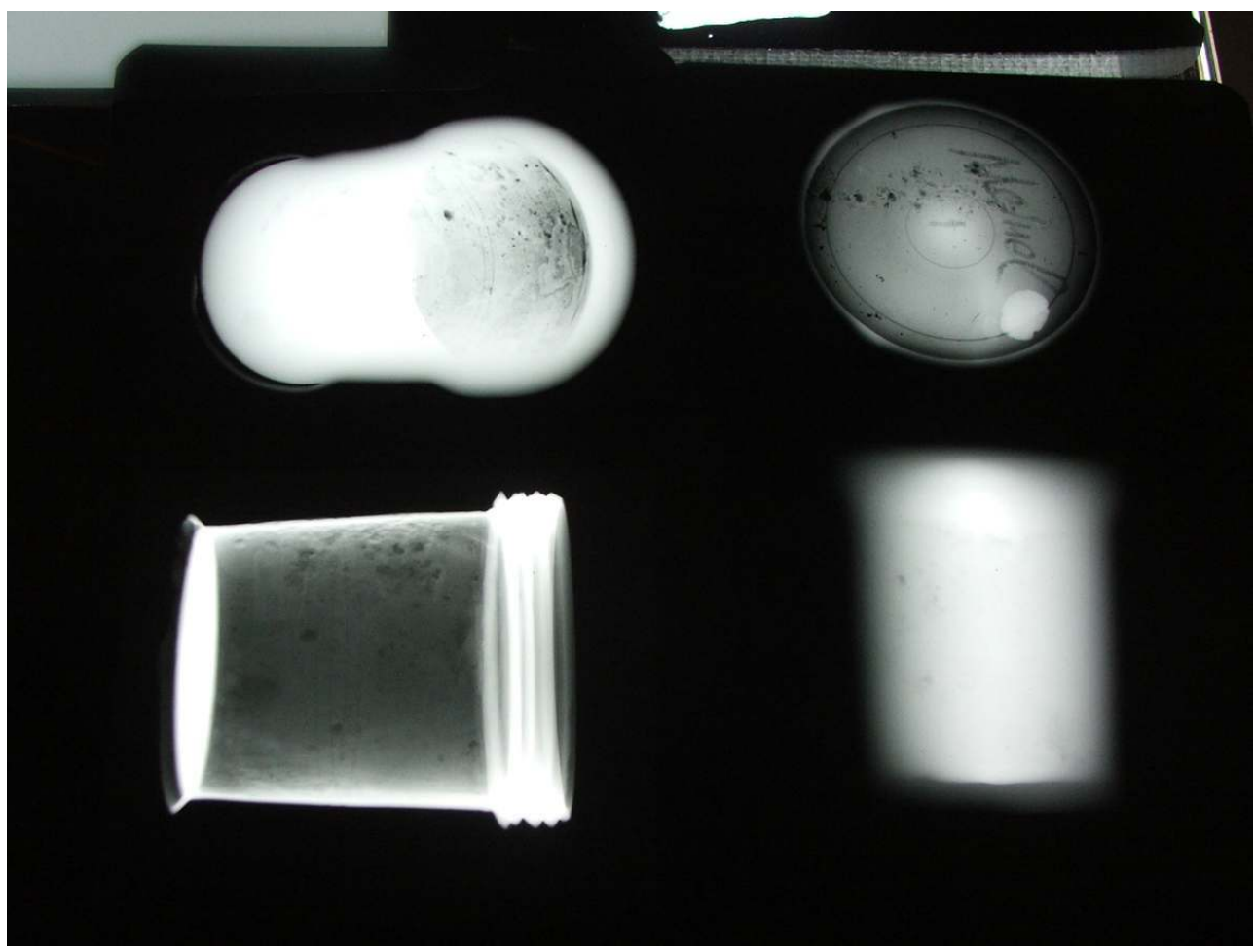

Tomographie d'une des boîtes en étain (c) C2RMF, 2006 
Figure 3

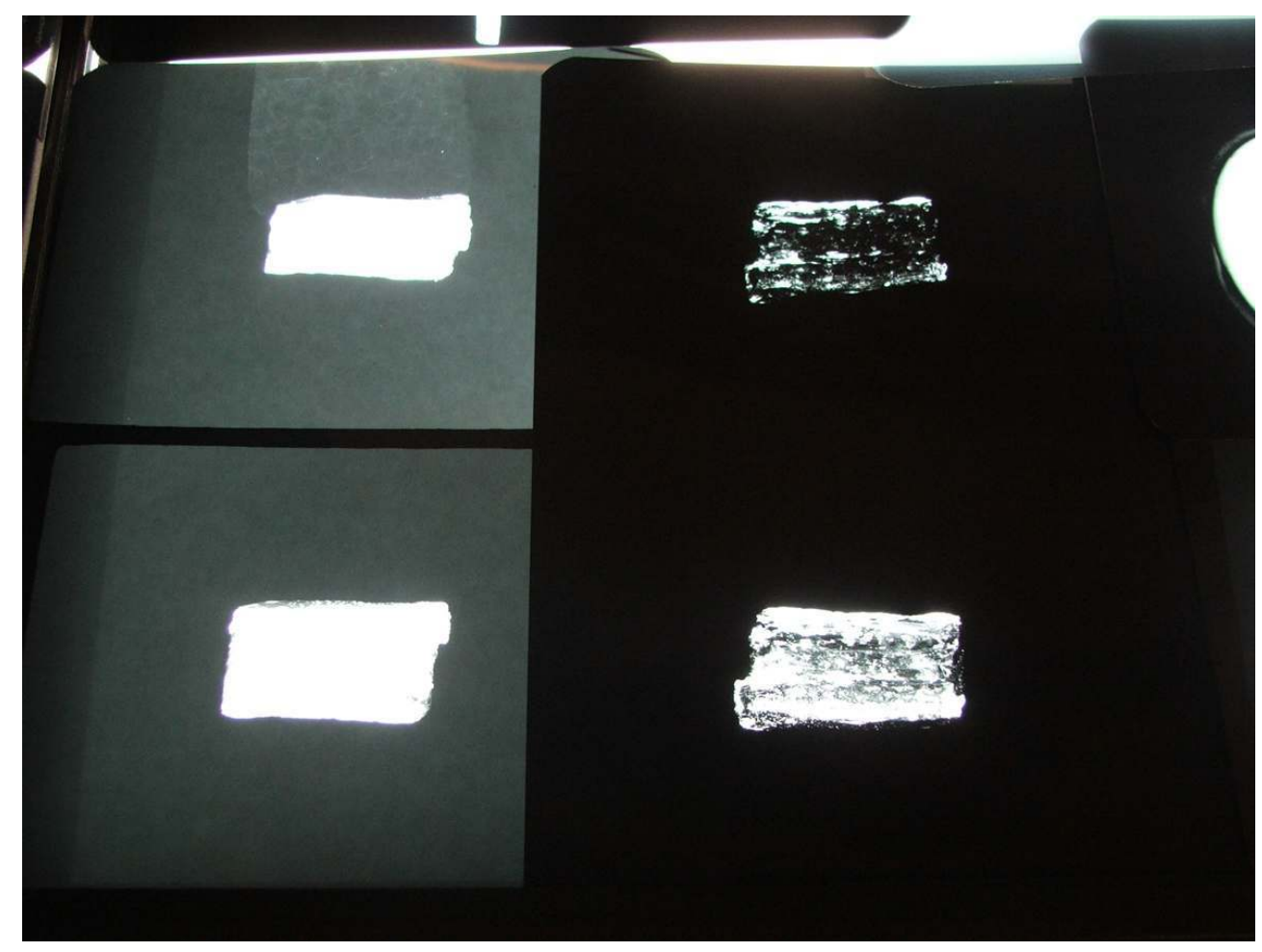

Tomographie d'une des boîtes en étain

(c) C2RMF, 2006

$8 \quad$ Afin de mener au mieux ce projet et avant de tenter un retrait des objets non identifiés de leur conditionnement, ils ont été examinés. Grâce au soutien du Centre de recherche et de restauration des musées de France (C2RMF), des spécialistes ont réalisé une tomographie ${ }^{5}$ et une microfluorescence des matériaux. Ces prospections avaient pour objectifs de déterminer leur composition, la lisibilité ou non d'un tracé qui aurait subsisté, l'observation de la structure interne des conditionnements et donc d'affirmer ou d'infirmer le choix d'une tentative d'extraction. (fig. $n^{\circ} 1, n^{\circ} 2, n^{\circ} 3$ ) 


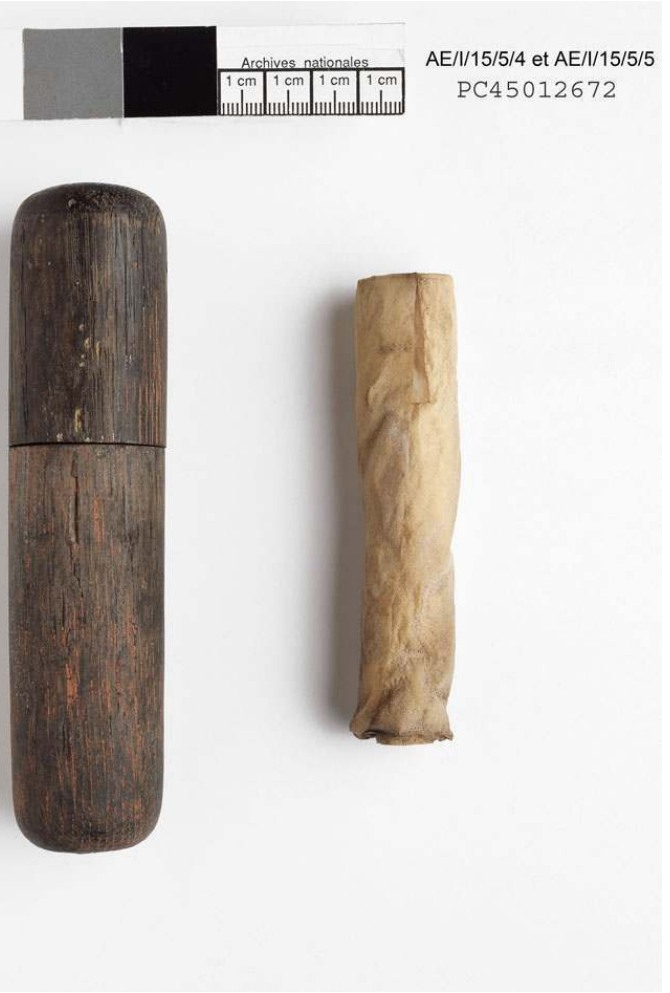

La boîte $n^{\circ} 7$ renfermant un parchemin déshydraté

Atelier de photographies, 2006. (c) Archives nationales

\section{Interventions sur le rouleau de parchemin contenu dans le petit étui de bois trouvé dans le bras du cavalier de bronze}

9 La boîte $n^{\circ} 7$ a livré un rouleau de parchemin visiblement en assez bon état de conservation mais dont la teneur intellectuelle n'était pas connue ni déchiffrable en l'état. Nul doute que cet étui en bois, fermé par un capuchon, a joué le rôle de tampon face aux variations climatiques qui se sont produites à l'intérieur de la statue. Le rouleau de parchemin présentait essentiellement un état de déshydratation important qui empêchait sa manipulation et interdisait toute tentative de déroulement qui risquait de l'abîmer. La nature du parchemin en fait un matériau particulièrement solide mais dont la grande faiblesse réside dans sa sensibilité à l'hygrométrie. Par conséquent, la seule façon de l'assouplir, étape indispensable avant de tenter de le dérouler, était de le placer dans une atmosphère humide contrôlée afin qu'il absorbe le plus lentement possible les molécules d'eau alors en quantité insuffisante. (fig. $n^{\circ} 5, n^{\circ} 6, n^{\circ} 7, n^{\circ} 8, n^{\circ} 9$ ) 
Figure 5

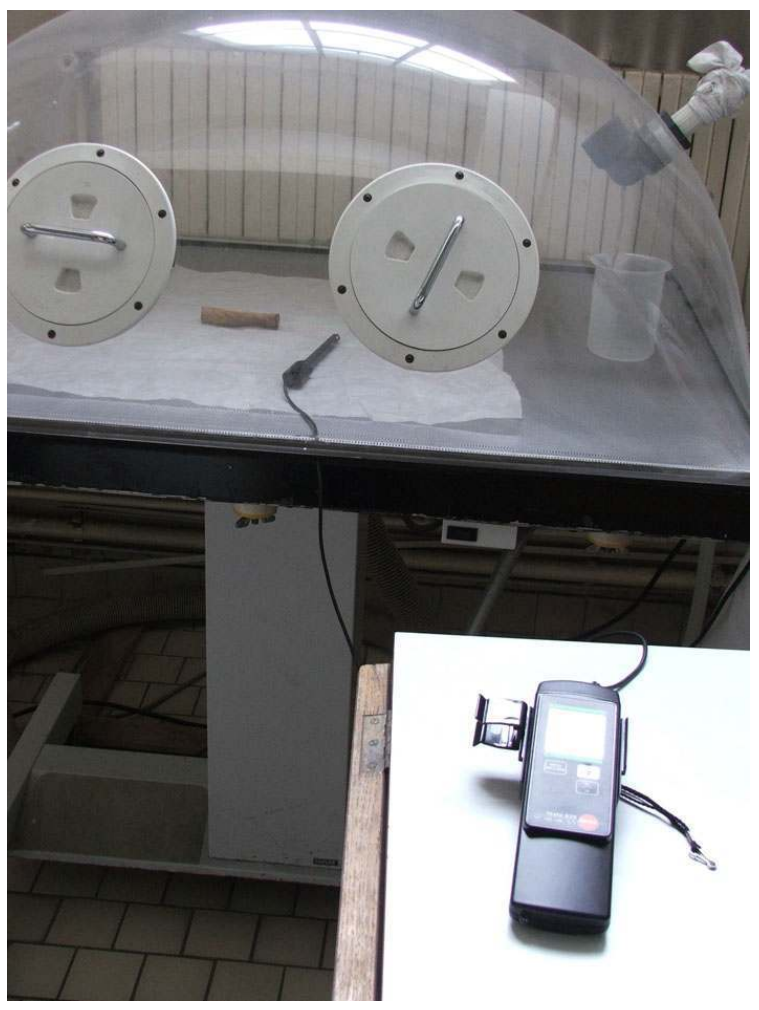

Réhydratation progressive en chambre humide

Phot. Martin-Saint-Clair, Laurent, 2006. (c) Archives nationales 
Figure 6

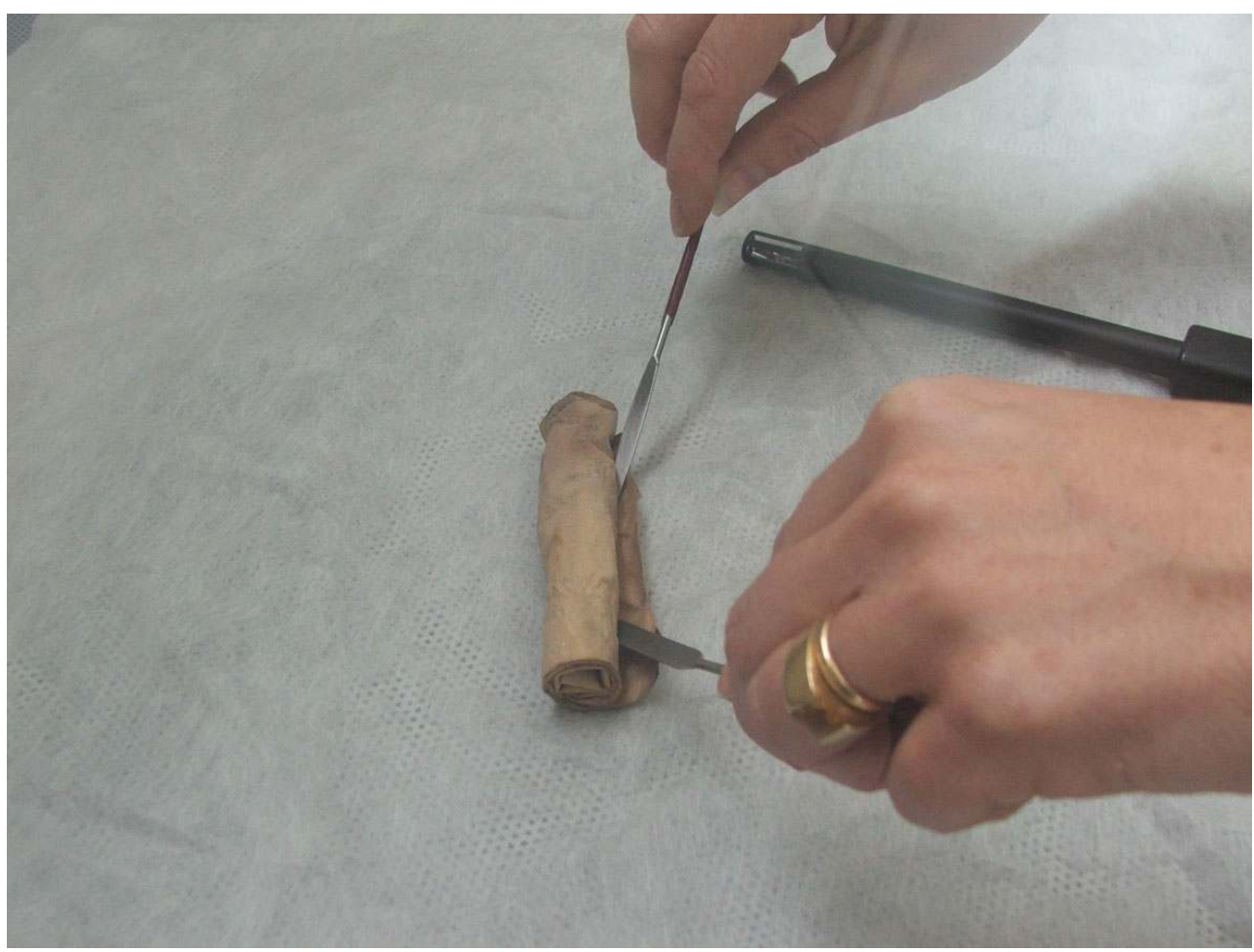

Ouverture après plusieurs heures sous le dôme d'humidification à 80 \% d'humidité relative Phot. Martin-Saint-Clair, Laurent, 2006. (c) Archives nationales 
Figure 7

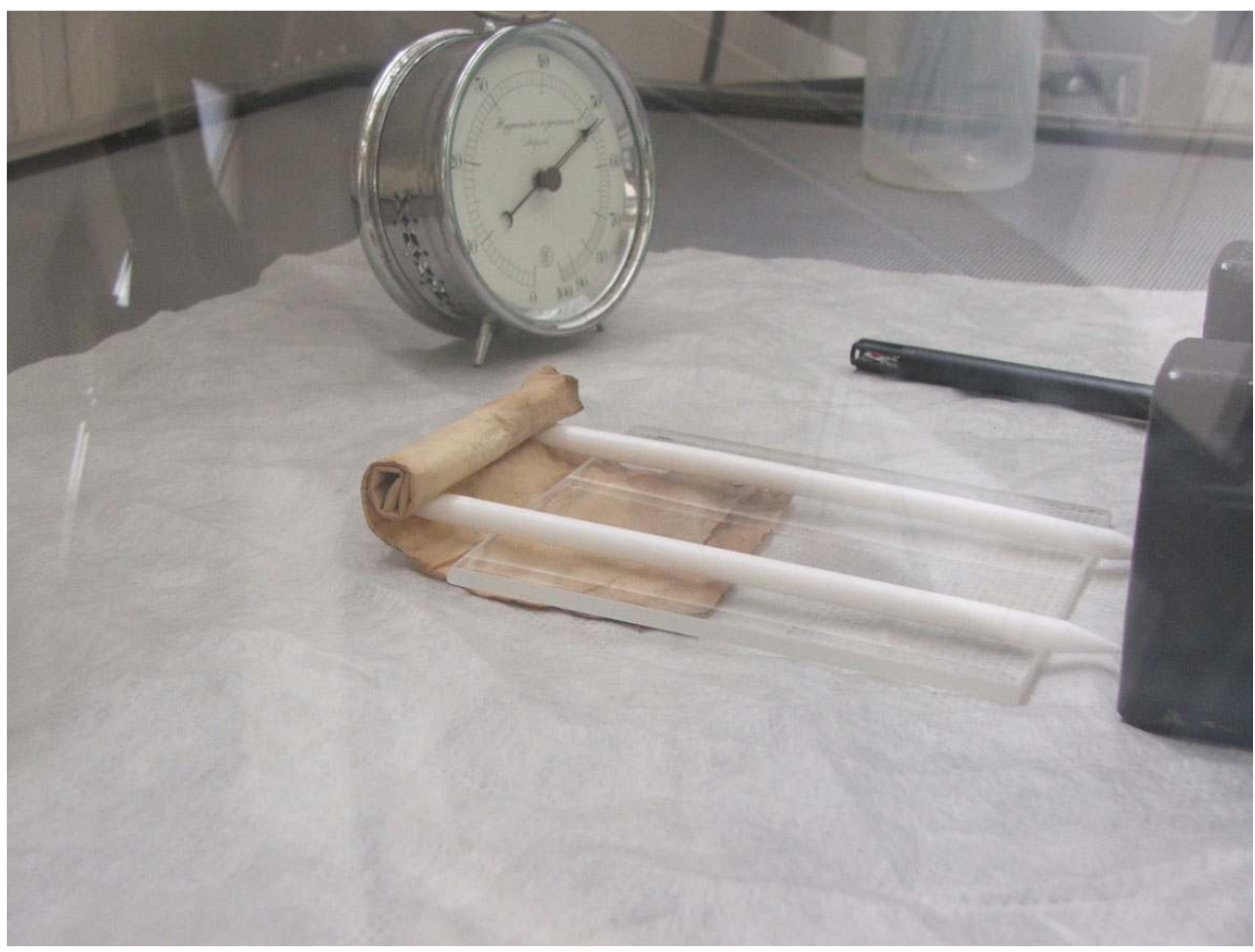

Mise en place de cales en téflon et de plaque d'altuglas@ pour aider à l'ouverture. Contrôle de l'humidité relative au sein de la chambre humide

Phot. Martin-Saint-Clair, Laurent, 2006. (c) Archives nationales 
Figure 8

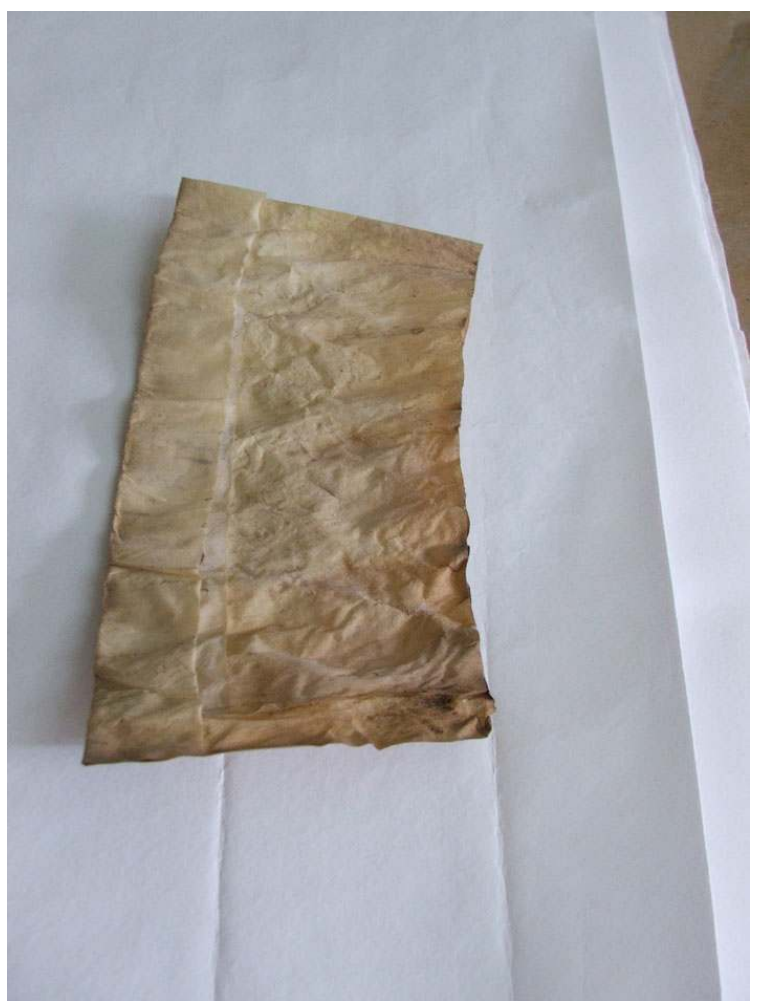

Le parchemin déroulé mais dont la planéité n'était pas suffisante pour permettre la lisibilité des tracés Phot. Martin-Saint-Clair, Laurent, 2006. (c) Archives nationales 
Figure 9

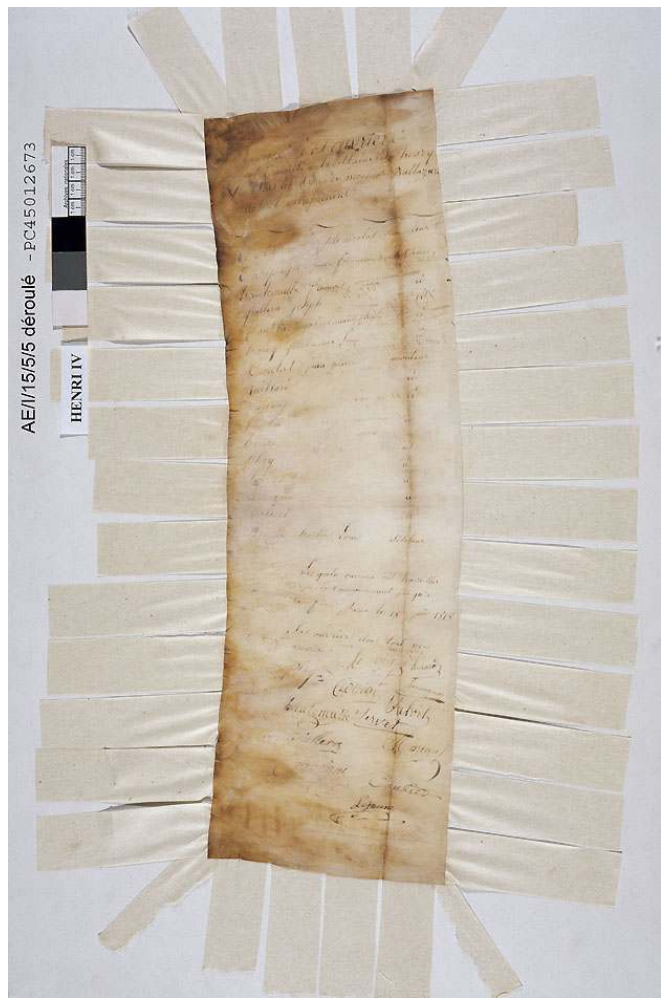

Mise sous poids entre des feuilles de gore-tex@ pour améliorer la planéité du support Phot. Martin-Saint-Clair, Laurent, 2006. (c) Archives nationales 
Figure 10

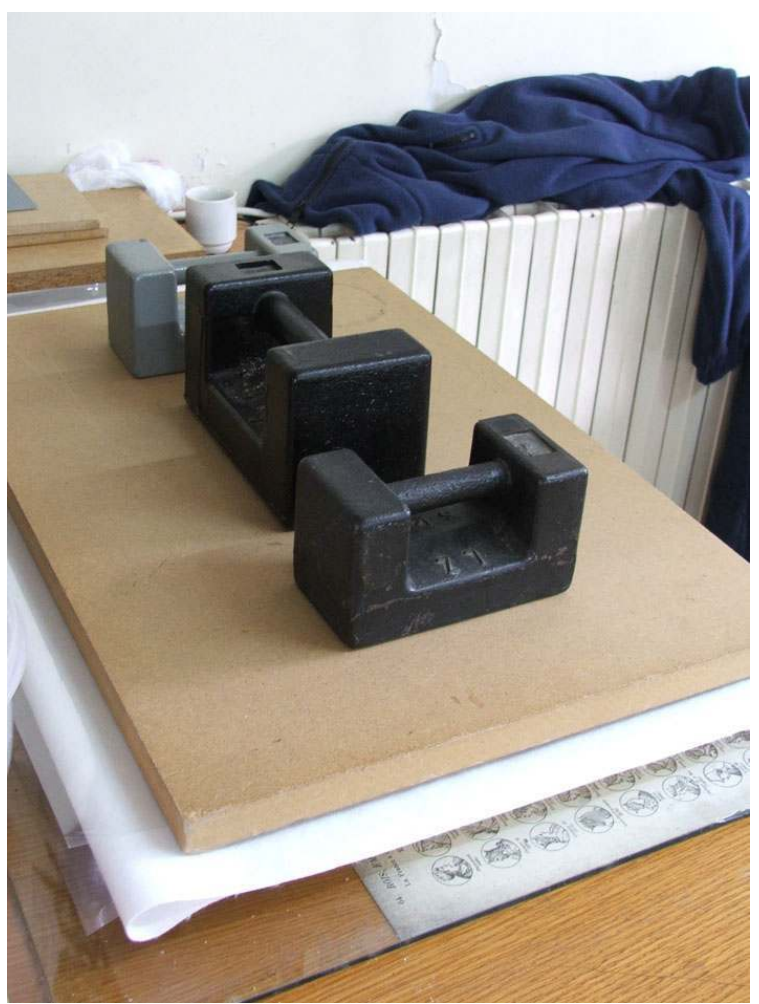

Le parchemin déroulé et déplié révélant la liste des ouvriers participant à la construction de la statue. Sur le bord gauche la zone de déformation qui était du côté de l'ouverture de l'étui est visible. Préparation des tirants en papier japonais

Phot. Martin-Saint-Clair, Laurent, 2006. ㄷ Archives nationales

Les restaurateurs ${ }^{6}$ ont ensuite pu procéder à l'observation de l'ensemble du document. Sur le parchemin déplié était maintenant lisible la liste des fondeurs et ciseleurs qui avaient participé à la construction de la statue de Henri IV. Mais ce document resté si longtemps roulé et dans des conditions de conservation défavorables, ne pouvait pas être conditionné tel quel dans une boîte d'archives. Pour maintenir le parchemin à plat, les restaurateurs ont donc conçu un montage sur un support rigide en polycarbonate cannelé et l'ont maintenu à l'aide de tirants en papier japonais (Kozo) collés à la colle d'amidon. En cas de variations thermo-hygrométriques et si les contraintes mécaniques et les tensions internes sont trop grandes, ils joueront le rôle de ressorts et céderont avant le parchemin, évitant ainsi de nouvelles déchirures ou déformations. (fig. $\left.\mathbf{n}^{\circ}{ }^{10}\right)\left(\right.$ (fig. $\left.\mathbf{n}^{\circ}{ }^{10}\right)$

11 Aujourd'hui, le document est conservé sur son support dans une boîte de conservation réalisée sur mesures par l'atelier et dont les matériaux répondent aux normes de longue conservation.

\section{Interventions sur les deux reliures agglomérées. Les Économies Royales de Sully}


Figure 11

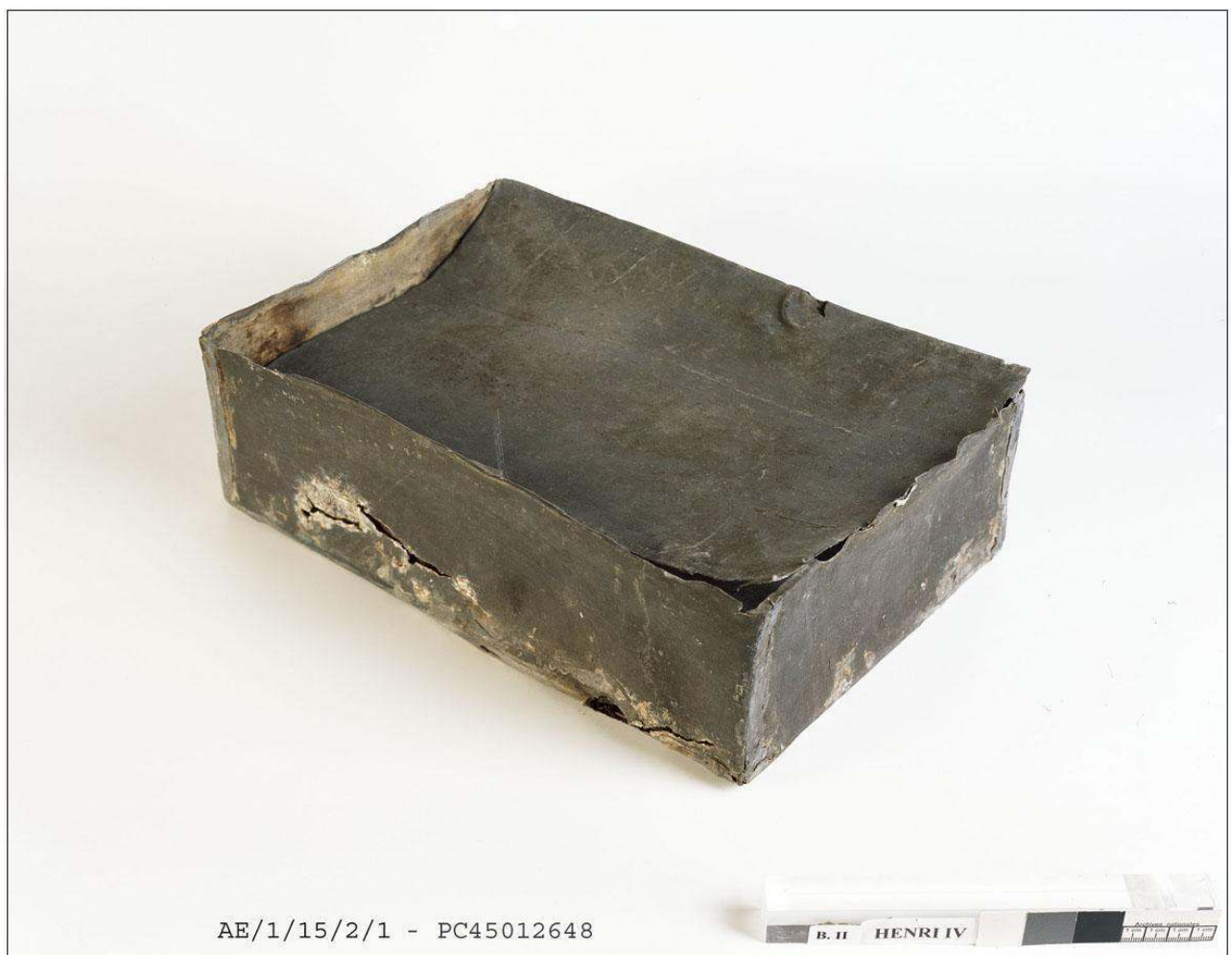

La boîte $\mathrm{n}^{\circ} 2$ renfermant Les Économies Royales

Atelier de photographies, 2006. (c) Archives nationales 
Figure 12

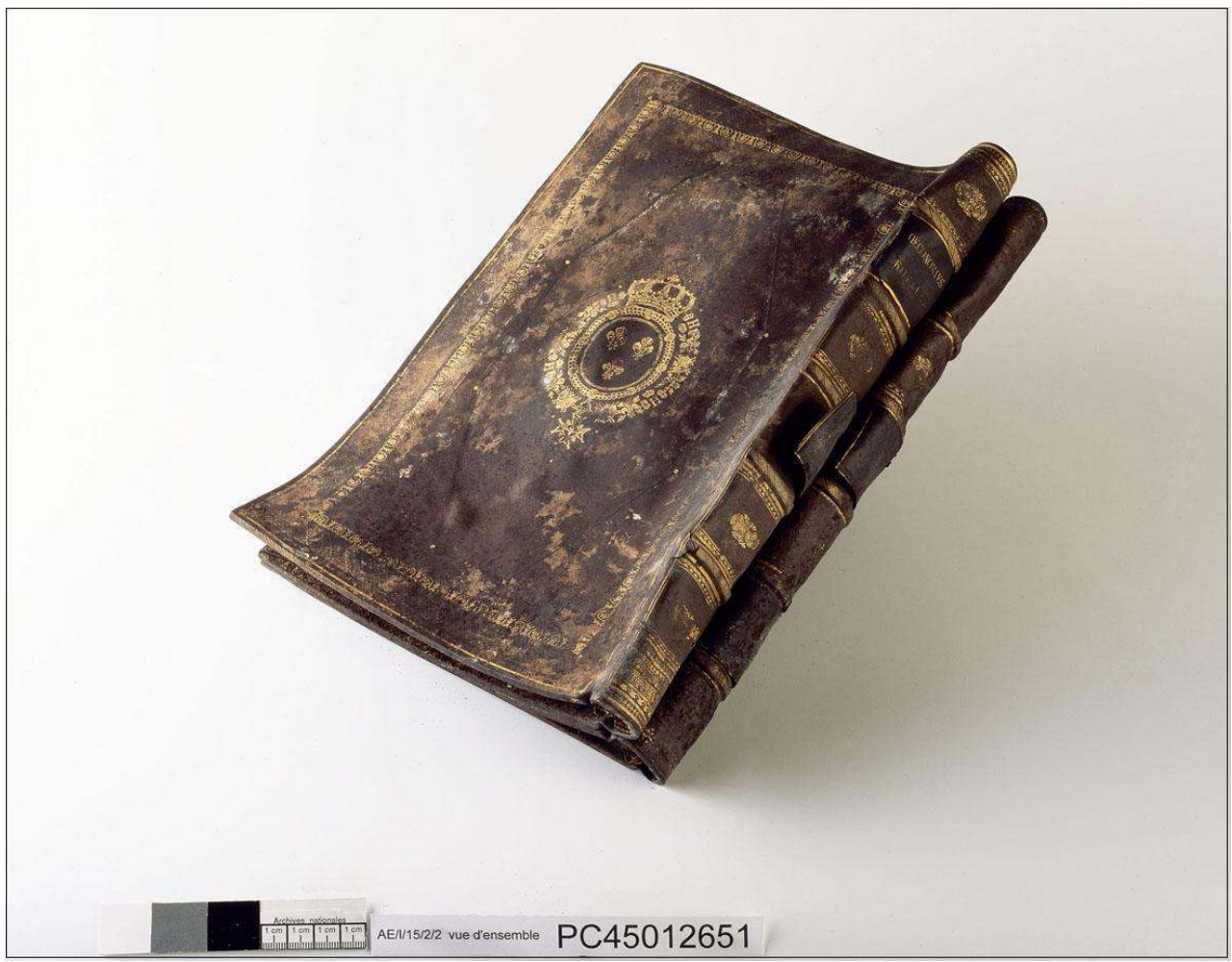

Les plats décorés des deux reliures Les Économies royales de Sully Atelier de photographies, 2006. (c) Archives nationales 


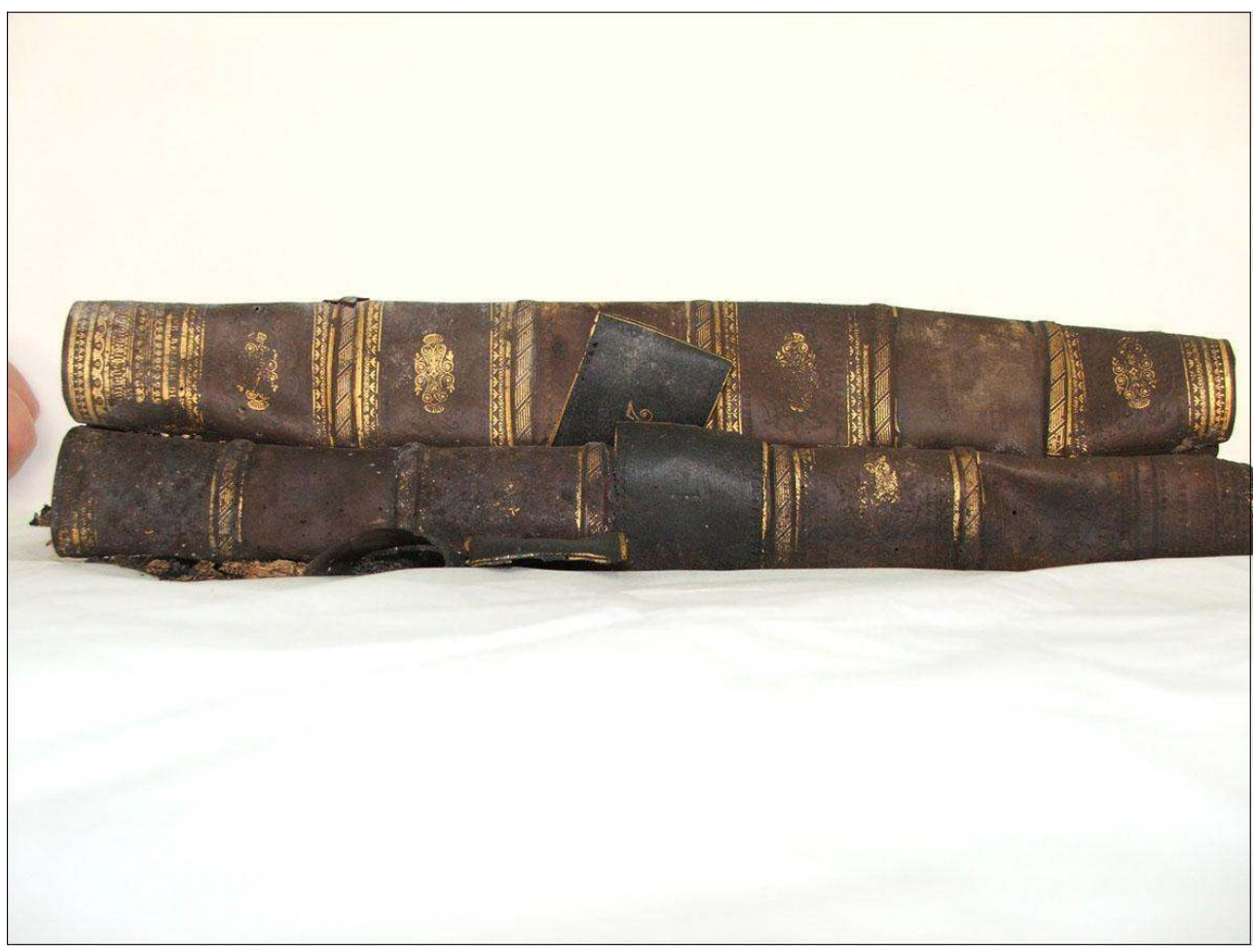

Les deux reliures agglomérées et déformées

Atelier de photographies, 2006. (c) Archives nationales

12 Les Économies Royales de Sully découvertes dans la boîte $\mathrm{n}^{\circ} 2$ présentaient un état de dégradation très préoccupant. En effet, le plat inférieur de la première reliure et le plat supérieur de la seconde se sont agglomérés entre eux, ne formant plus qu'un seul bloc rigide et très fragile. Du corps d'ouvrage du premier volume, il ne reste qu'une matière informe très compacte. Du second, il ne reste que de la poussière de papier. L'essentiel du travail de restauration ${ }^{7}$ a consisté à nettoyer le cuir des reliures et à consolider les parties fragiles, notamment les pièces de titre qui ont été refixées au dos des reliures par un soufflet de papier japonais. (fig. $n^{\circ} 14, n^{\circ} 15, n^{\circ} 16, n^{\circ} 17, n^{\circ} 18, n^{\circ} 19, n^{\circ} 20$ ) 
Figure 14

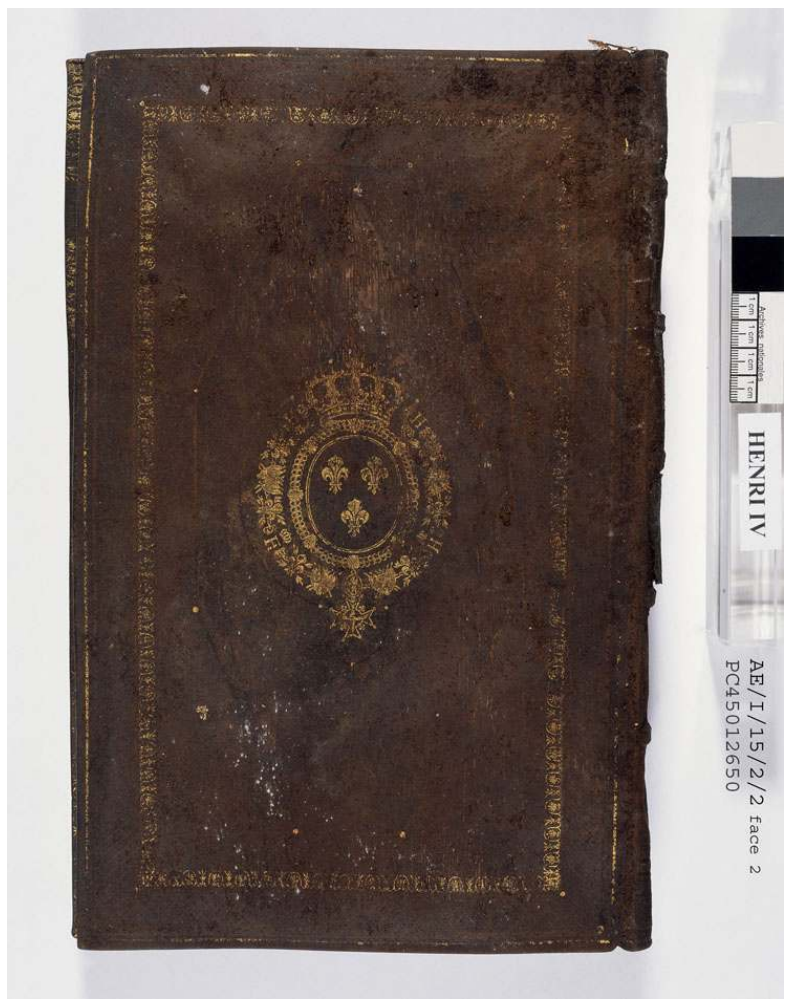

Plat avant nettoyage à sec

Atelier de photographies, 2006. (C) Archives nationales 
Figure 15

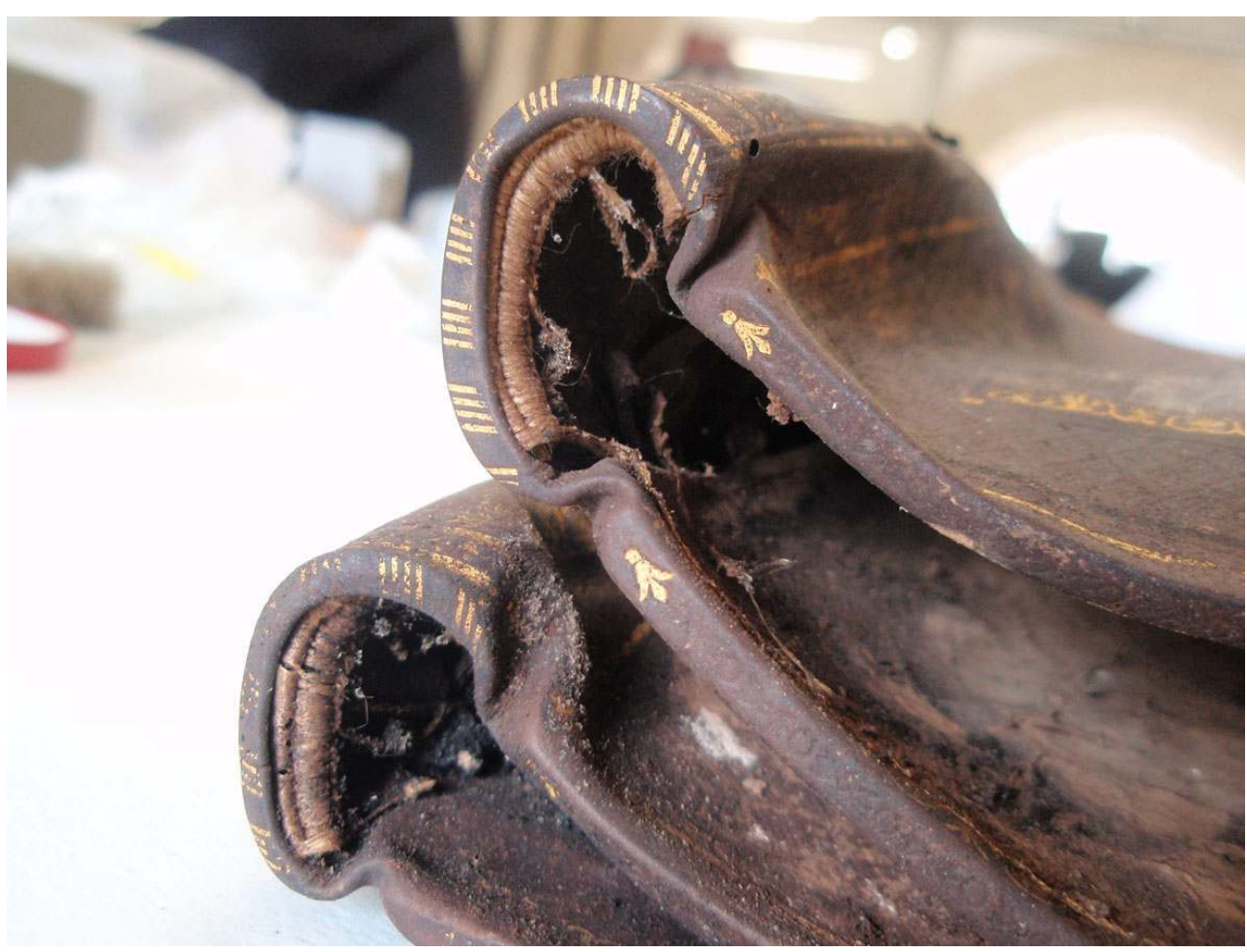

Vue des dos agglomérés

Phot. Coste, Patricia, 2006. (c) Archives nationales 
Figure 16

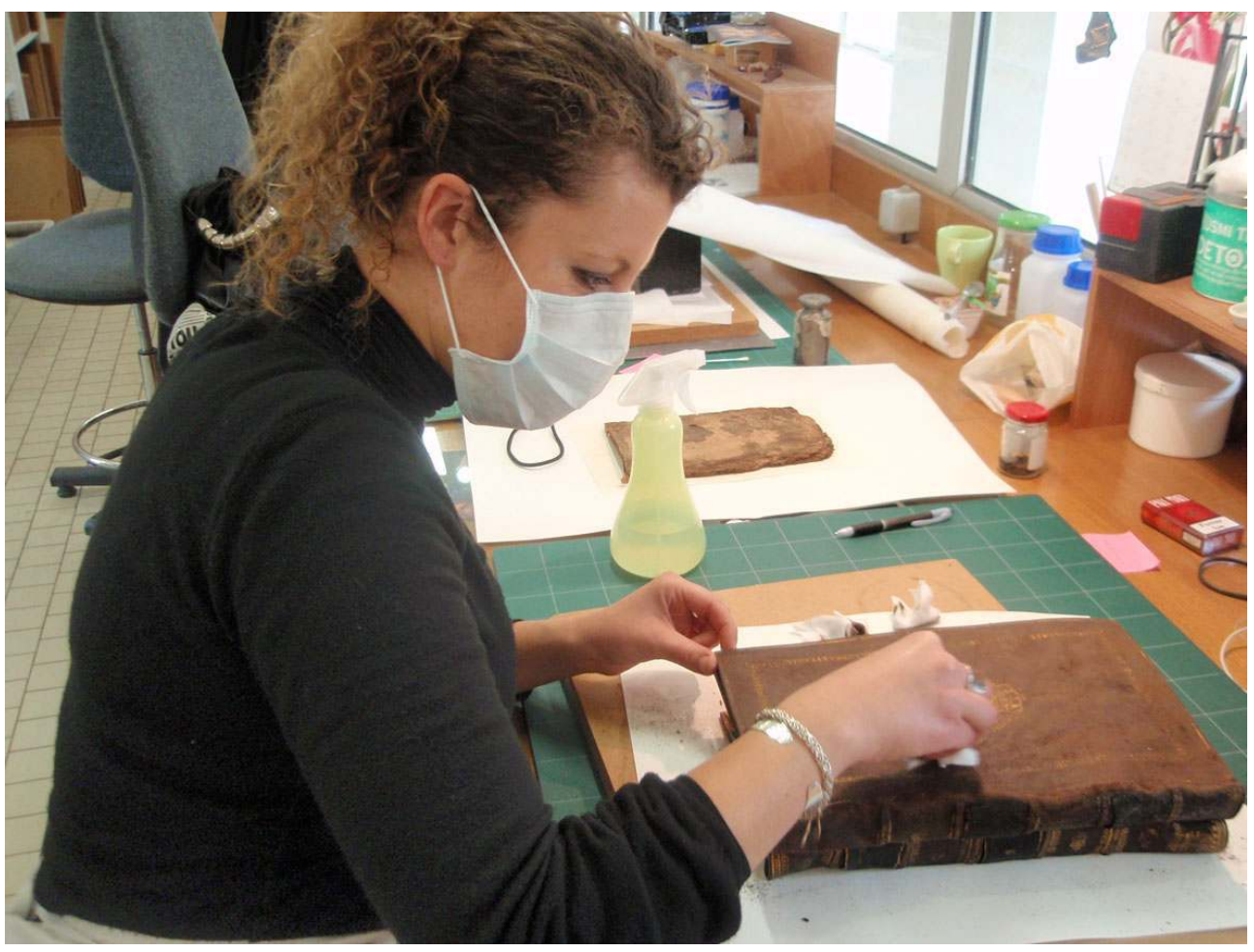

Les reliures en cours de nettoyage

Phot. Coste, Patricia, 2006. (c) Archives nationales 


\section{Figure 17}

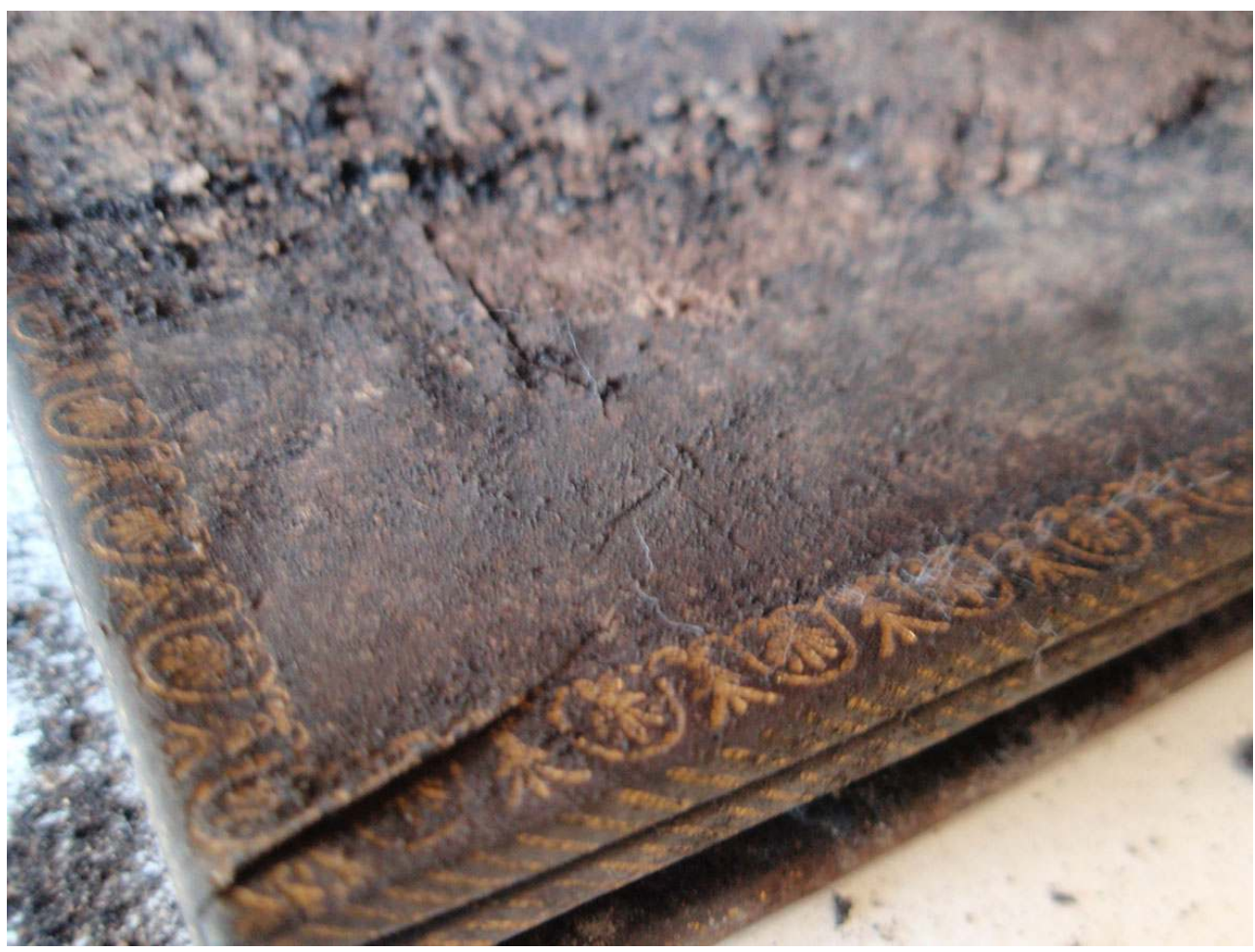

Le contre-plat avant nettoyage

Phot. Coste, Patricia, 2006. (c) Archives nationales 
Figure 18

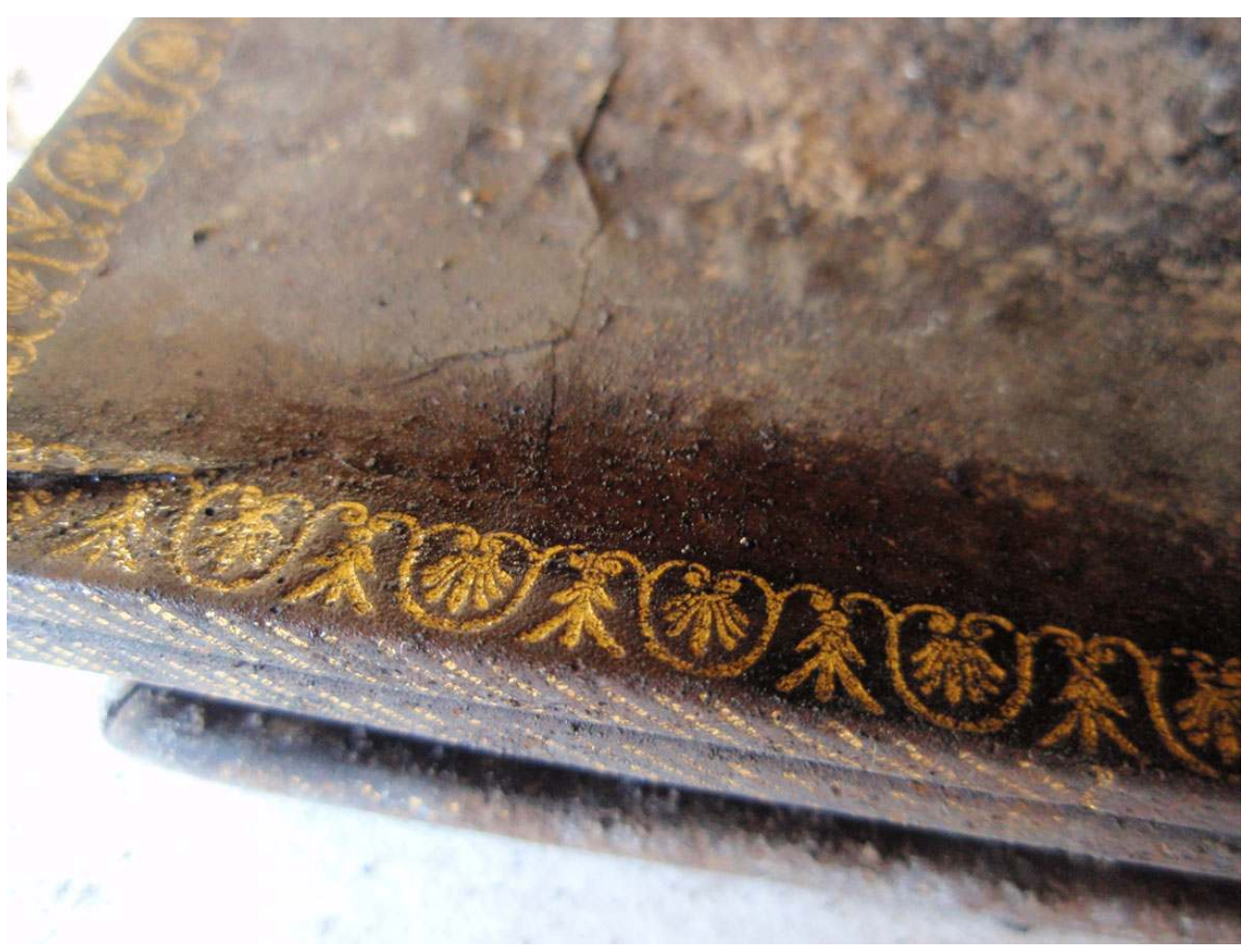

Le contre-plat après nettoyage

Phot. Coste, Patricia, 2006. ( ) Archives nationales 


\section{Figure 19}

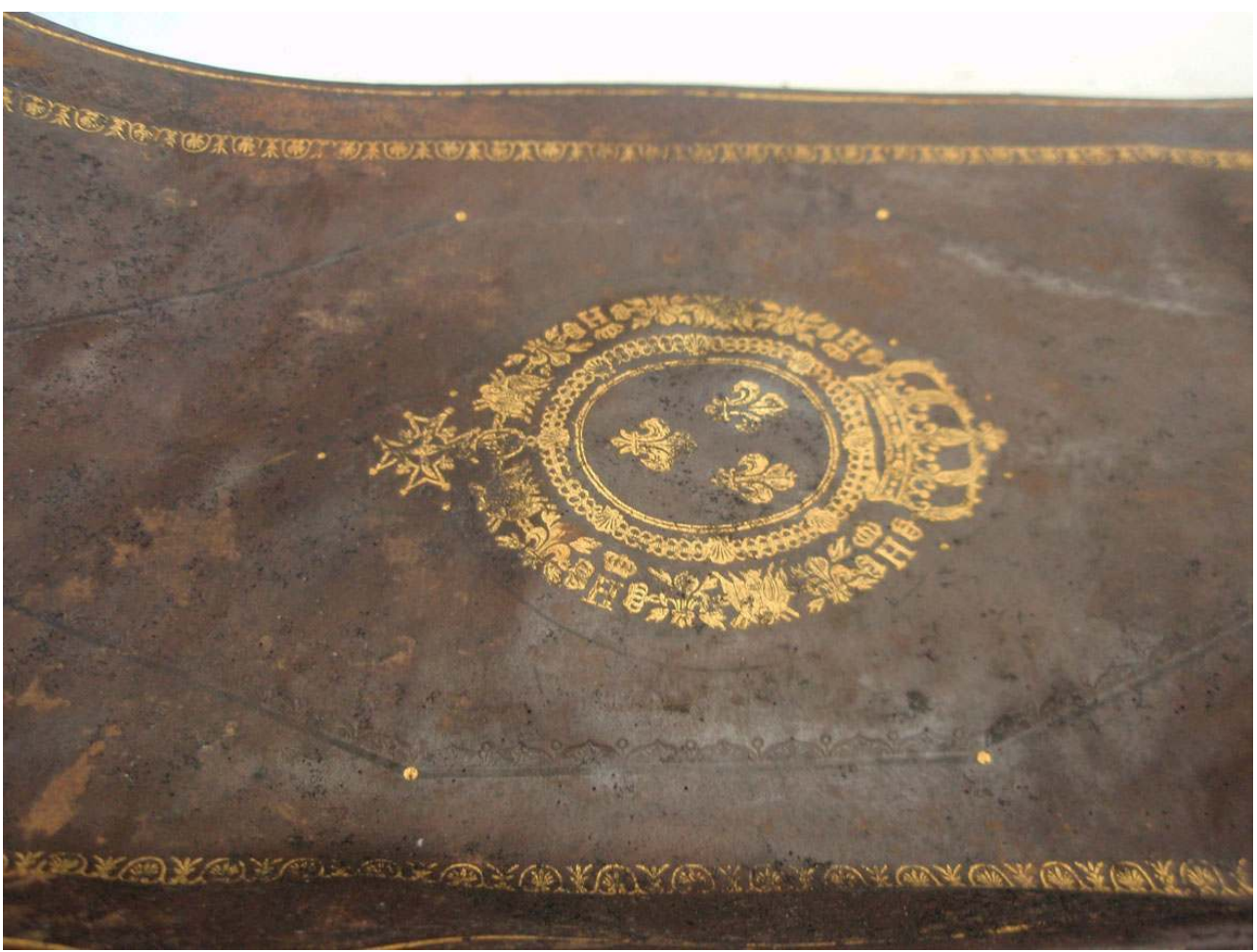

Plat supérieur avant/après nettoyage

Phot. Coste, Patricia, 2006. (c) Archives nationales 
Figure 20

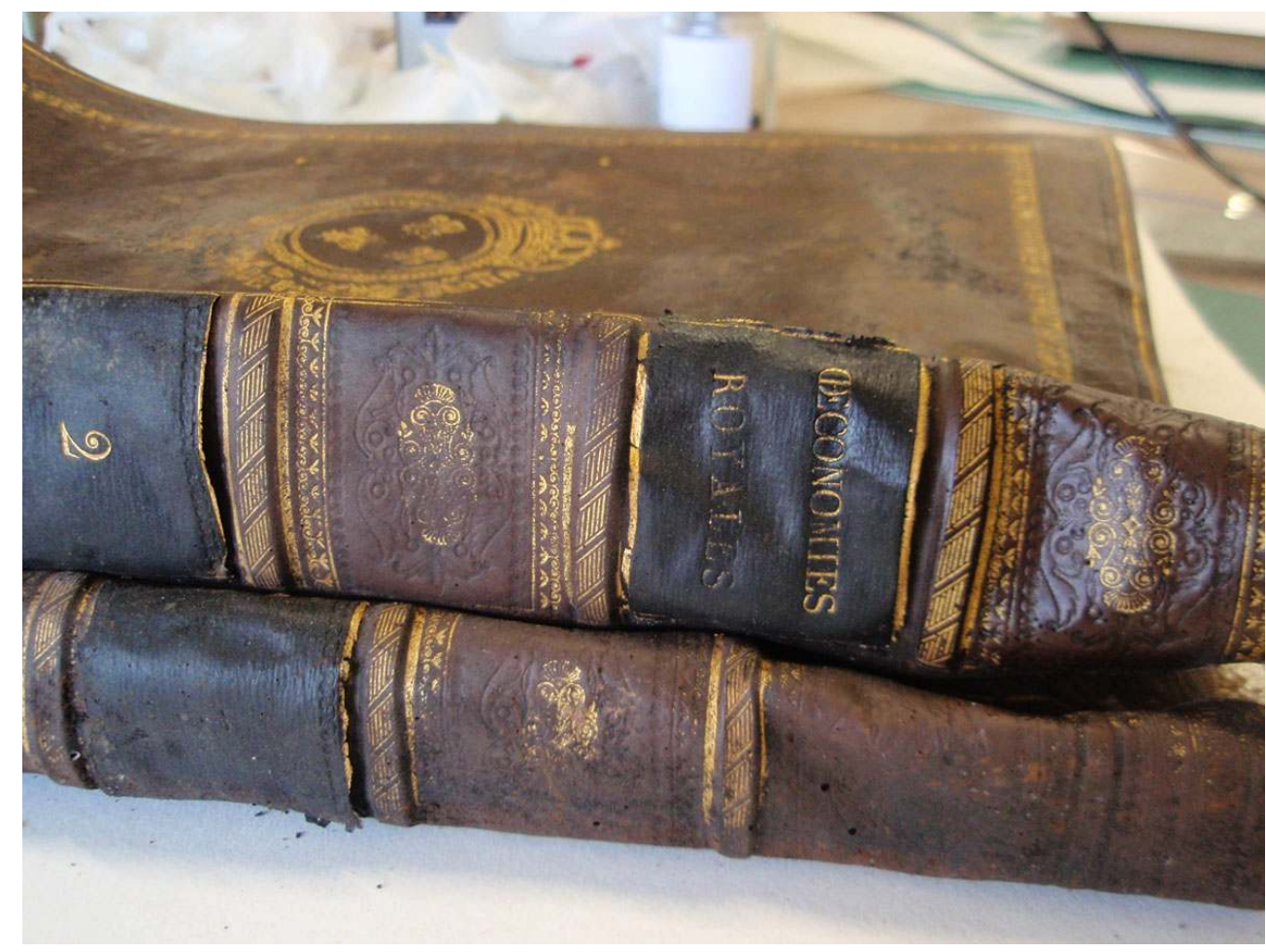

Les deux reliures après nettoyage

Phot. Coste, Patricia, 2006. ( ) Archives nationales

\section{Focus sur une restauration : La reliure Histoire de Henri IV le grand, édité en 1816 chez Antoine Augustin Renouard trouvée dans la boîte $n^{\circ} 4$ et fabriquée dans les ateliers Simier}

ERIC LAFOREST, Technicien d'art restaurateur-relieur de l'atelier de restauration de documents graphiques, de reliures et de dorures des Archives nationales

René Simier fonda son atelier de reliure à Paris, rue Saint-Honoré, vraisemblablement entre 1780 et 1792. Son travail était connu de la noblesse émigrée, puis sous l'Empire, de l'Impératrice Marie-Louise qui appréciait particulièrement ses reliures.

En 1816, deux ans après la Restauration, dans l'année où l'Histoire du Roi Henri le Grand fut éditée à Paris chez Renouard, Louis XVIII le nomma au poste prestigieux de « Relieur du Roi ».

16 C'est à un artisan talentueux et « bien en cour » (malgré son activité professionnelle sous l'Empire) qu'on fit appel pour inclure symboliquement une reliure monarchiste dans le socle d'une statue célébrant le retour de la royauté.

17 Alphonse, le fils de René Simier, lui aussi «Relieur du Roi » sous Charles X et Louis Philippe, ainsi que les successeurs de Simier, pérennisèrent la notoriété de ce grand nom de l'artisanat du livre jusqu'à M. Jean-François Barbance et ses fils (restaurateurs de livres et doreurs sur cuir actuellement encore en fonction). 

partie des volumes issus des ateliers de prestige durant la première moitié du XIX ${ }^{e}$ siècle (on peut citer par exemple les ateliers Thouvenin, Trautz-Beauzonnet-Purgold, contemporains des Simier.) Ces caractéristiques propres se traduisent par un soin extrême apporté au choix et à la variété des matériaux constitutifs, une qualité d'exécution et de finition notamment dans les décors souvent irréprochables mais aussi dans la volonté stylistique et professionnelle de marquer son temps. rigueur : la précision apportée au façonnage de ces reliures, jusque dans les aspects les moins visibles du corps d'ouvrage, pose la principale problématique du restaurateur ${ }^{8}$ qui prend en charge un tel volume : en conserver la «fraîcheur » et tenter de lui préserver autant que possible son authenticité esthétique, ne pas l'alourdir, donc l'appauvrir par une intervention trop visible. Cependant, conserver l'authenticité n'est pas dissimuler à outrance le travail de restauration. Cet équilibre, reste un problème délicat toujours débattu et à résoudre au cas par cas. Le technicien en charge de cette reliure se trouve donc face à un artisanat "moderne " de très haute facture, d'une grande précision technique, qu'il convient, sinon d'imiter, du moins de comprendre, afin d'être plus efficace dans les interventions de restauration. être à l'origine de quelques difficultés auxquelles peut s'attendre le restaurateur dans ses interventions. couvrure et la parure des cuirs rendront les soulèvements et les déposes difficiles voire impossibles. Puis, certaines opérations de transformation subies par les matériaux: le glaçage, le gaufrage, les marbrures acides des cuirs et des papiers, ainsi que le couchage ou d'autres traitements des feuillets en vogue au XIX ${ }^{\mathrm{e}}$ siècle (azurage et blanchiment), qui rendront certaines manipulations en milieu humide hasardeuses.

dorures fournies et exécutées avec un souci de perfection certain au XIX ${ }^{\mathrm{e}}$ siècle ont, quant à elles, l'inconvénient de réduire les marges de manœuvre lors d'opérations sur les cuirs. ainsi que la multiplication des mises en presse et des apprêtures de cahiers, rendent souvent ces volumes extrêmement fragiles car très rigides. par celui-ci) qui orientait forcément la mise en œuvre du travail, cet objet présentait évidemment une très grande valeur historique due au contexte de l'inauguration de la statue de Henri IV, doublée d'une valeur esthétique transmise par Simier, sans parler de l'aspect purement bibliophilique, scientifique ou intellectuel du contenu.

Aussi, une des nécessités du travail du restaurateur a été de prendre connaissance de ces risques, de ces "valeurs ", de les hiérarchiser, s'il le pouvait, à l'aide de tous les acteurs en charge du document, et ainsi, avec l'expérience, d'avoir un regard plus précis sur l'objet et de sécuriser au mieux les opérations de restauration. 


\section{Constat d'état général du volume}

27 À l'examen visuel, on a pu relever une grande différence entre d'une part, le bon état général de conservation extérieure, en particulier celui des matériaux de recouvrement du volume et, d'autre part, l'état de dégradation mécanique et biologique interne.

Le bon état de conservation, hormis quelques traces ténues de moisissures, du grain long vert, des tranches dorées à la feuille, des décors dorés au blanc d'œuf réalisés à la roulette et constitués de filets, des tranchefiles plates de soie, du signet, etc., est surprenant si l'on prend en considération le mode de conservation des objets contenus dans le socle, et a fortiori ceux contenus dans la tête et le bras du cavalier de bronze. (fig. $\mathbf{n}^{\circ} \mathbf{2 1}, \mathbf{n}^{\circ} \mathbf{2 2}$ )

Figure 21

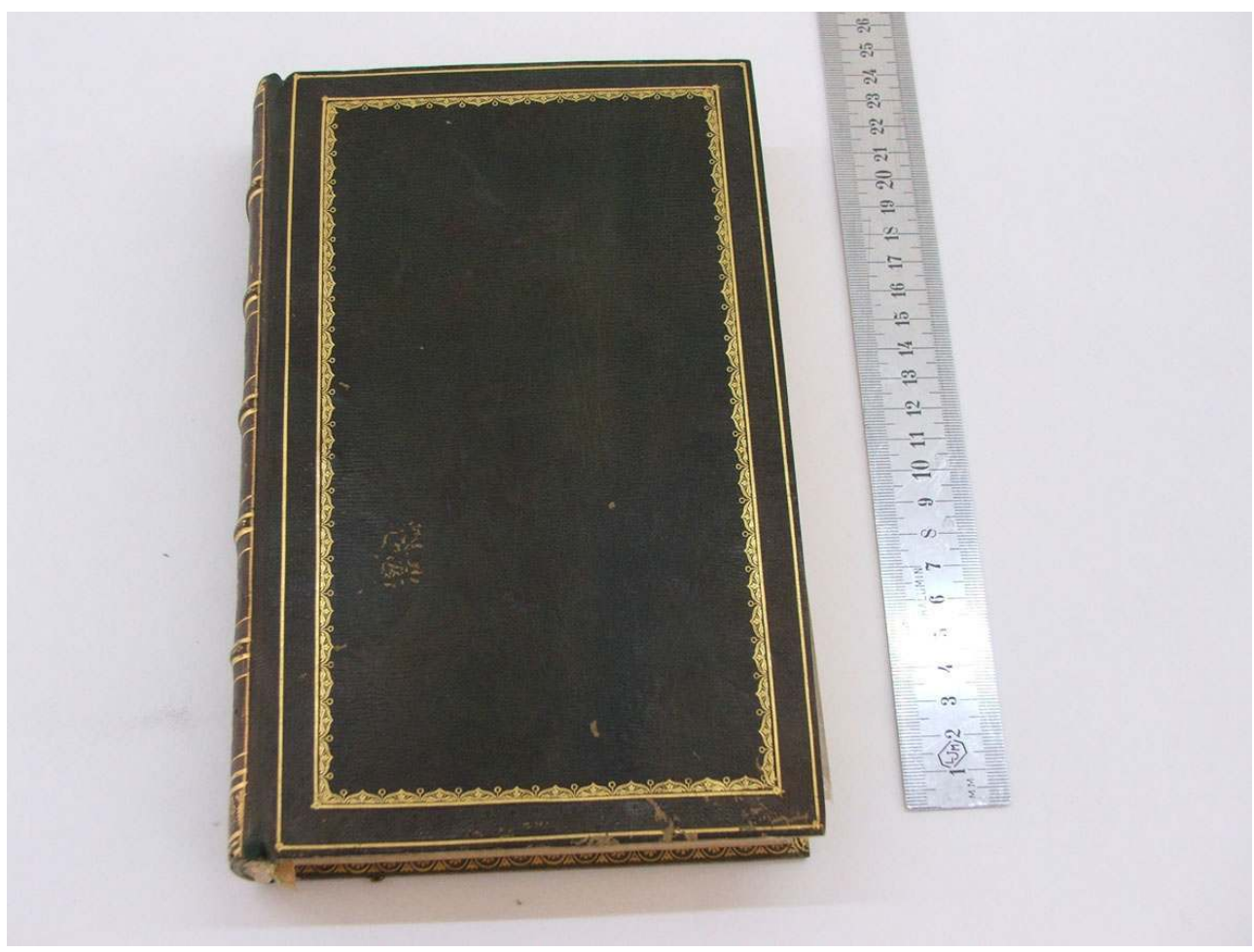

Vue externe du volume

Phot. Laforest, Éric, 2006. (c) Archives nationales 


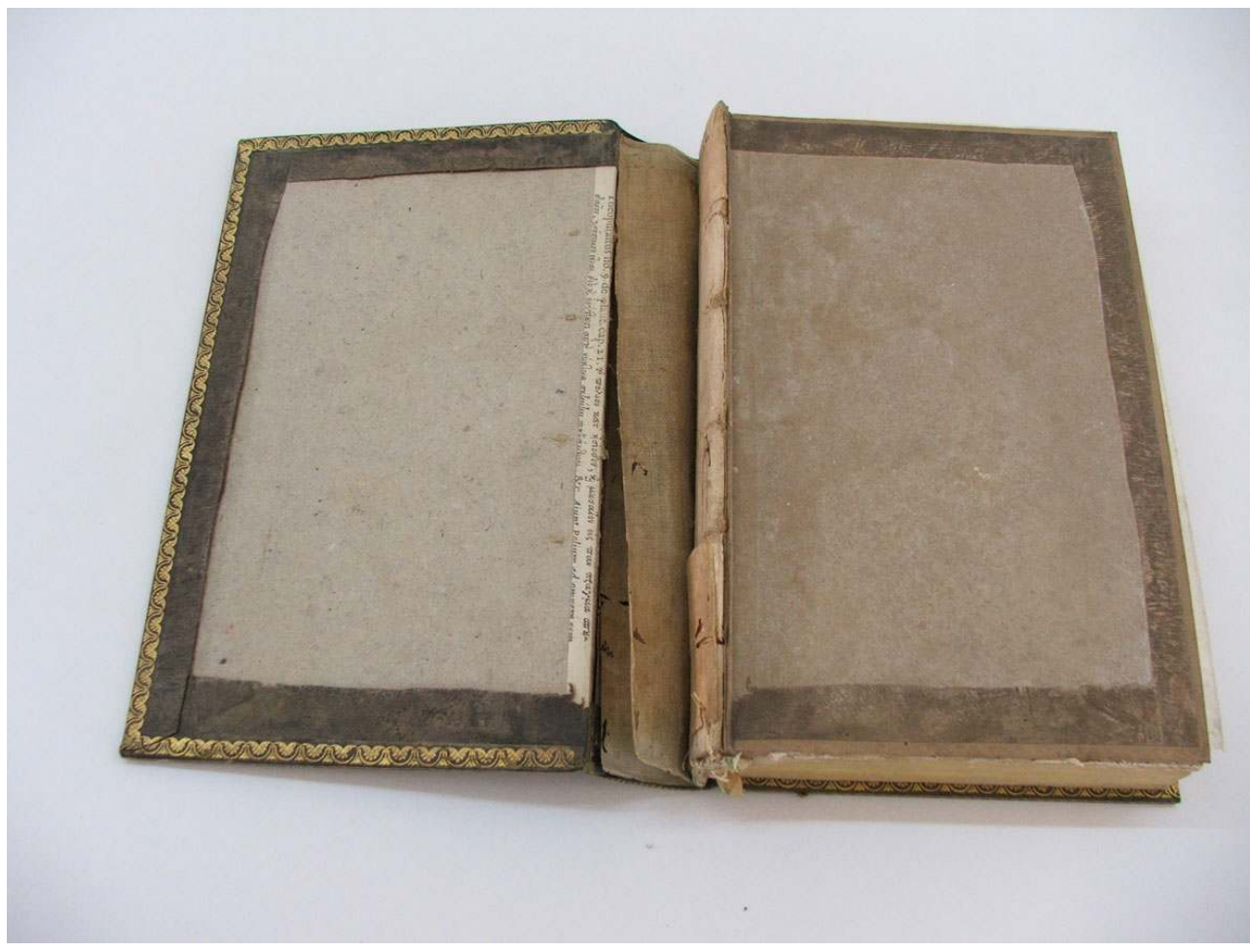

Vue interne du volume

Phot. Laforest, Éric, 2006. (C) Archives nationales

En effet, il suffit d'examiner en comparaison certains des documents conservés à proximité de cette reliure et qui n'ont pas eu la chance d'être épargnés par les attaques climatiques et bactériologiques comme les deux volumes de Sully qui sont réduits à l'état de quasi poussière, ou le rouleau de parchemin.

De manière générale, il a été surprenant de constater l'état disparate de tous ces objets en fonction de la qualité de leur contenant et peut-être de la place réservée à ceux-ci dans le socle ou dans la tête de l'animal depuis près de 200 ans.

Les quelques dommages constatés sur le volume ont pour causes principales une forte exposition à l'humidité et des chocs thermiques plus que vraisemblables pour une conservation presque « de plein air » et de bord de Seine. Le peu de traces de moisissures consécutives, tant sur le cuir que sur le papier du bloc-texte, est étonnant dans ces conditions. Cependant l'humidité a entraîné quelques faiblesses ou ruptures mécaniques dans certains matériaux constitutifs notamment sur le fil de couture, le papier des premiers cahiers, l'apprêture du dos, les charnières internes, les attaches, la partie interne des faux-nerfs, les gardes intérieures très touchées.

On a constaté également de nombreuses galeries d'insectes dans la partie inférieure des premiers cahiers, aux endroits où le papier était le plus fragilisé par l'humidité, et sans doute plus sensible à ce genre de dégradation. De même, aux endroits atteints par l'humidité puis par les spores, ces insectes ont très légèrement creusé le chagrin des plats supérieur et inférieur mais de façon très partielle. On peut supposer que le corps d'ouvrage typique du XIX ${ }^{e}$ siècle, c'est-à-dire très compact, a opposé une certaine résistance aux attaques d'insectes. (fig. $\mathrm{n}^{\circ} 23, \mathrm{n}^{\circ} \mathbf{2 4}$ ) 


\section{Figure 23}

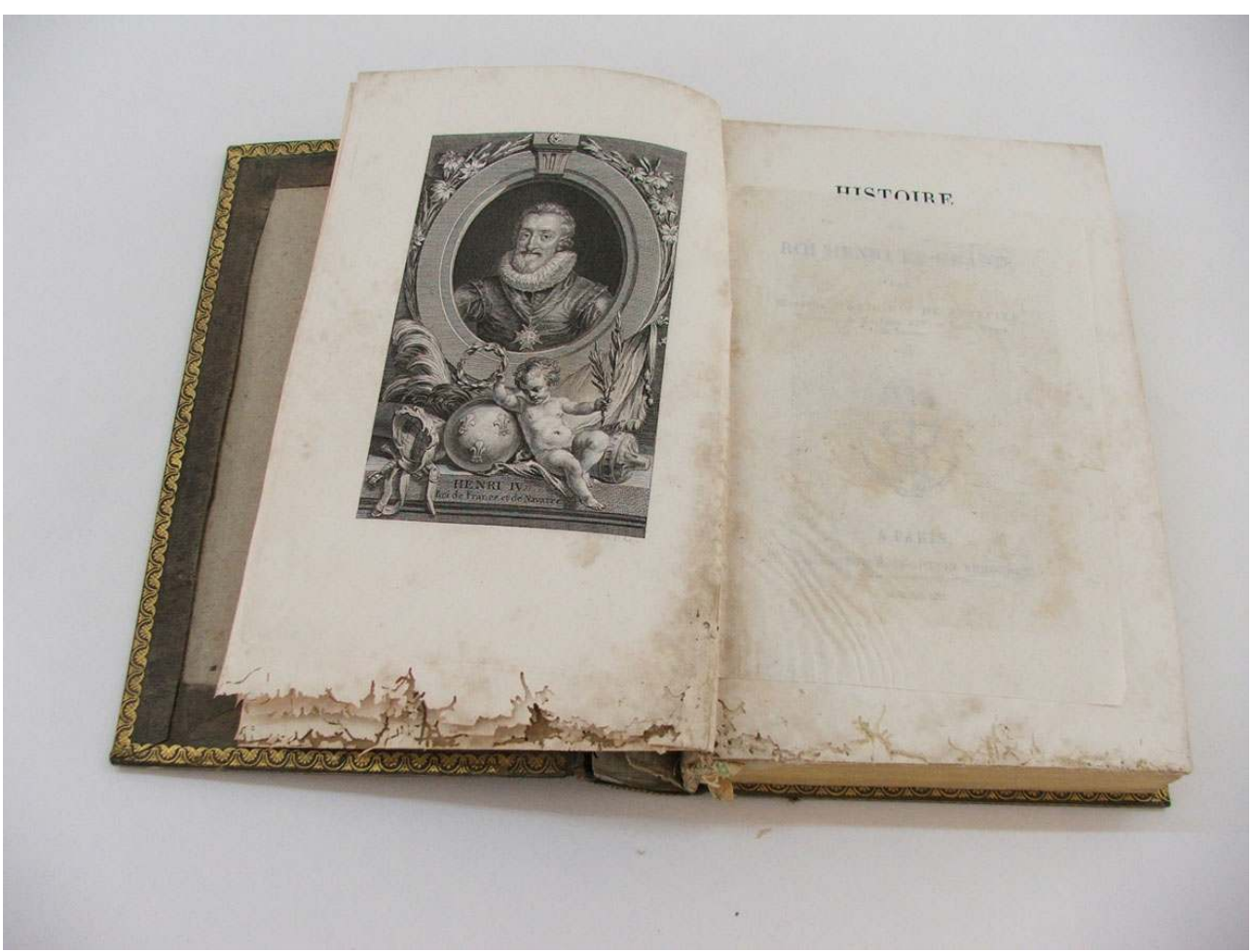

Le volume et les altérations du papier, détail des galeries

Phot. Laforest, Éric, 2006. (c) Archives nationales 
Figure 24

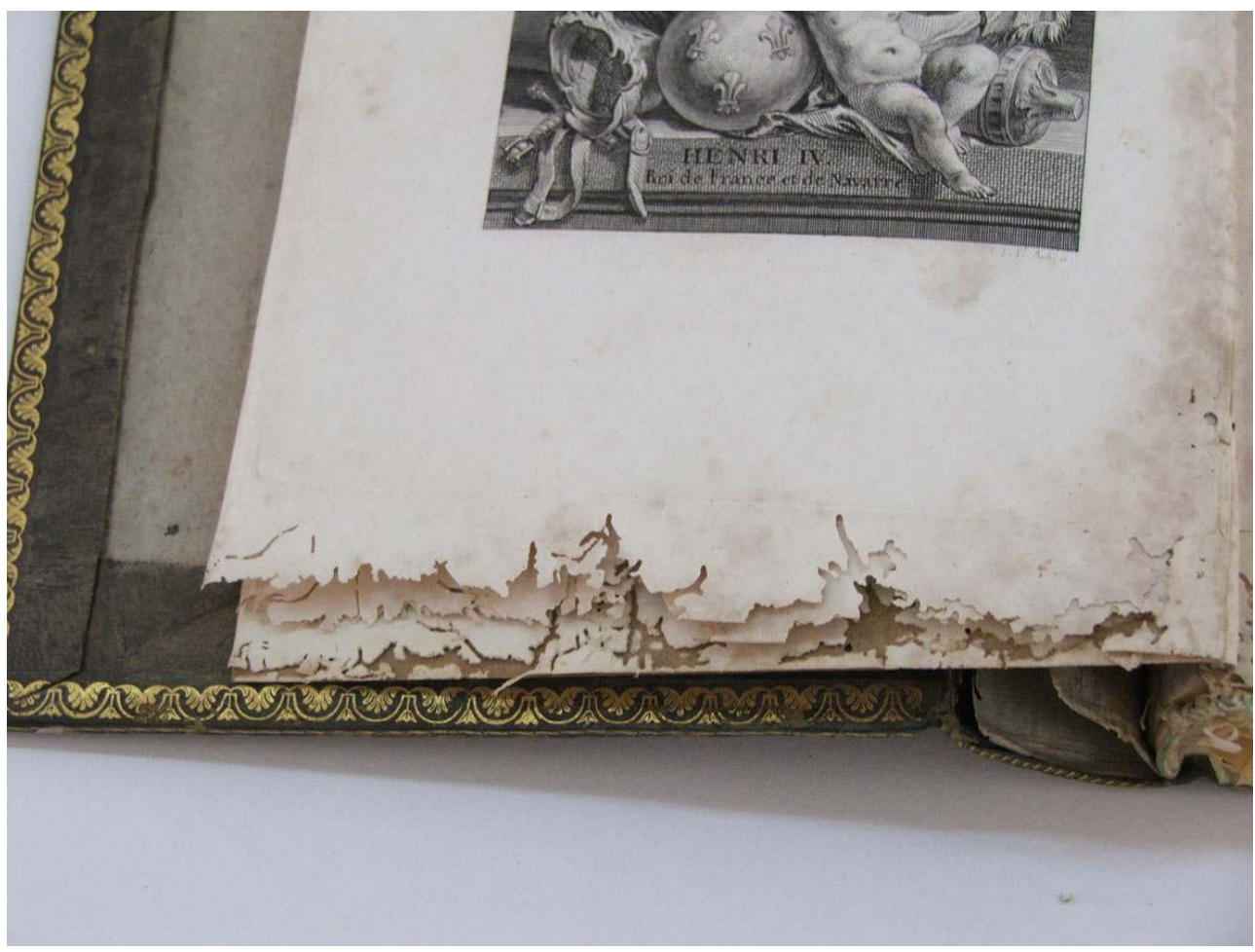

Le volume et les altérations du papier, détail des galeries

Phot. Laforest, Éric, 2006. (c) Archives nationales

\section{Examen et traitements}

Après le retour des documents envoyés en désinfection, il a été réalisé une restauration des dégradations essentiellement «mécaniques» du volume: ruptures, déchirures, lacunes, soulèvements et décollements.

Aussi, par ordre d'accessibilité des différentes parties, ont été déposées les gardes et contre-gardes, puis le premier cahier et le frontispice, enfin les différentes apprêtures et cartes de renfort du dos ainsi que les faux-nerfs (décollés par l'humidité). Ensuite on a procédé à la restauration du papier et à la restauration de la reliure.

\section{La restauration du papier}

Après un gommage à l'aide de gomme en poudre et un nettoyage "léger " par pulvérisation et tamponnage à l'eau et à l'éthanol, chaque feuillet déposé à été mis à plat par fond-tendu type karibari sur une plaque de plexiglas couverte d'une toile de polyester. Ces feuillets étaient maintenus sous tension à l'aide de tirants de papier japonais et de fausses-marges. Le comblement des lacunes a été réalisé par la réintégration bord à bord de papier japonais de même grammage que le papier d'origine. Les déchirures et les faiblesses du papier ont été consolidées à l'aide de papier japonais fin et transparent. Ces matériaux de consolidation dépassant du document servent à la fois de support à la réintégration définitive et de tirants pour le séchage. Ils forment une fausse marge de papier qui sera réhydratée avec le document et collée sur quelques 
millimètres sur le support. Il se produit alors une tension par évaporation naturelle et une remise à plat (selon la méthode japonaise adaptée du karibari). (fig. $\left.\mathbf{n}^{\circ} 25\right)$

Figure 25

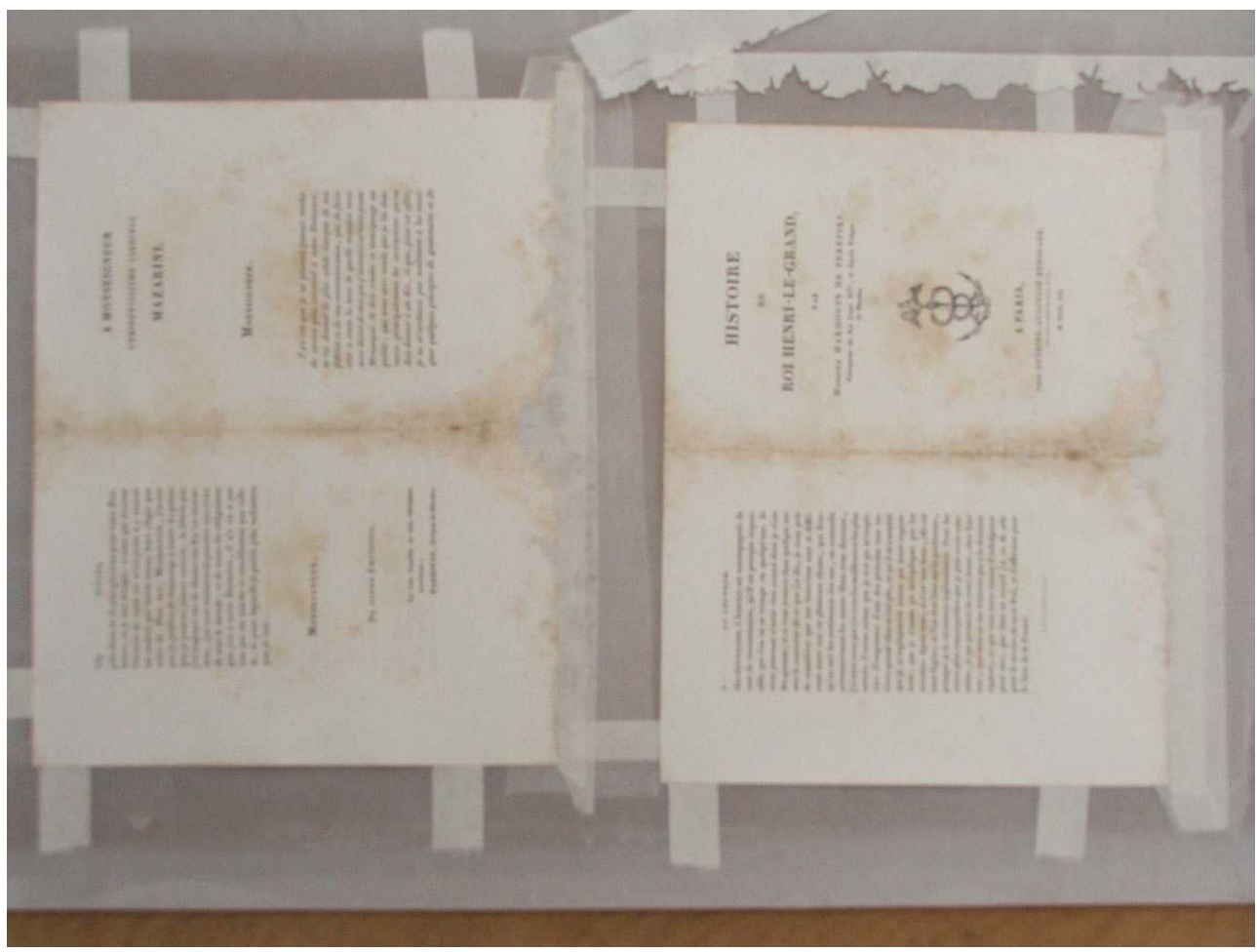

Le fond-tendu

Phot. Laforest, Éric, 2006. (c) Archives nationales

Cette méthode permet une mise en tension douce et efficace sans que le document ne soit jamais assujetti et contraint trop fortement par le «jeu» d'un fond-tendu traditionnel toujours aléatoire pour la remise à plat d'un papier fin. Il supprime, par là même, les mises sous presse qui altèrent les cuvettes des gravures et le grain du papier.

Le papier japonais est exclusivement en fibres naturelles de kozo peu raffinées et la colle fabriquée à partir d'amidon. La remise au ton des papiers de réintégration a été effectuée à l'aide d'aquarelle et de pastels secs.

\section{Les interventions sur le corps d'ouvrage et la reliure}

Le premier cahier a été reconstitué et cousu à son emplacement d'origine, la gravure réencartée. Les lacunes des cahiers suivants, moins atteints, ont été traitées localement par renforts de papier japonais collés en « sandwich».

Des apprêtures superposées du dos, seule la carte avec une mention manuscrite (Henri IV) a été conservée. Les autres cartes (acides) n'ont pas été réintégrées pour des raisons de conservation. Un papier japonais a été intercalé entre la carte d'origine et le dos des cahiers.

Les faux-nerfs ont été refixés à leur emplacement d'origine puis les attaches des plats, ainsi que la passure en carton, ont été reconstituées à l'aide de plusieurs minces fils de lin. 
41 Les gardes d'origine ont été reposées et les quelques lacunes du cuir sur les plats et les coiffes ont été comblées à l'aide de papier japon teinté.

42 Enfin, le volume à été très légèrement ciré à la cire 213 , dont une des propriétés est son action anti-fongique et de ne comporter aucun agent brillant qui empêche généralement le cuir de respirer dans son environnement. (fig. $n^{\circ} 26, n^{\circ} 27, n^{\circ} 28, n^{\circ} 29$ )

Figure 26

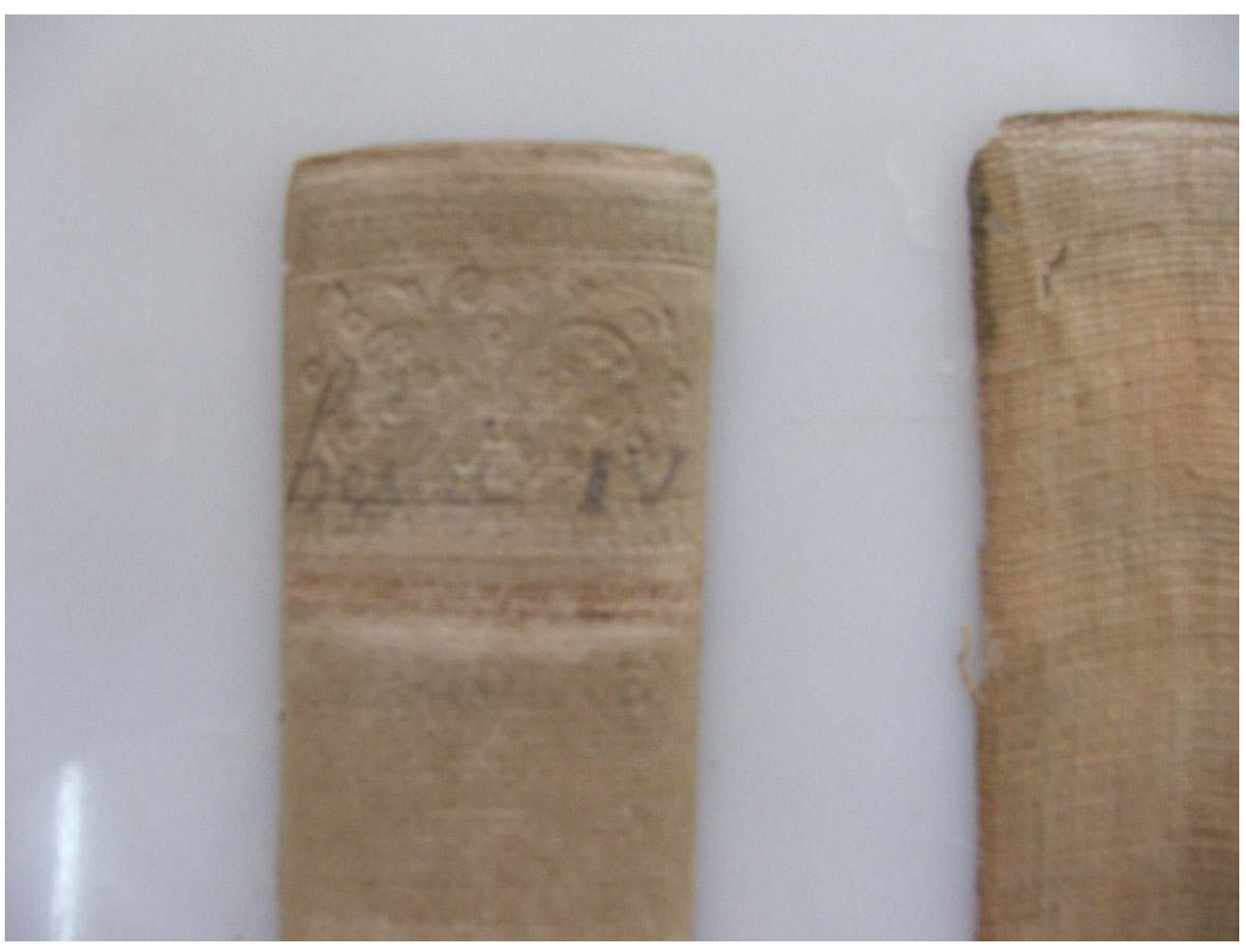

La carte avec la mention « Henri IV »

Phot. Laforest, Éric, 2006. @ Archives nationales 
Figure 27

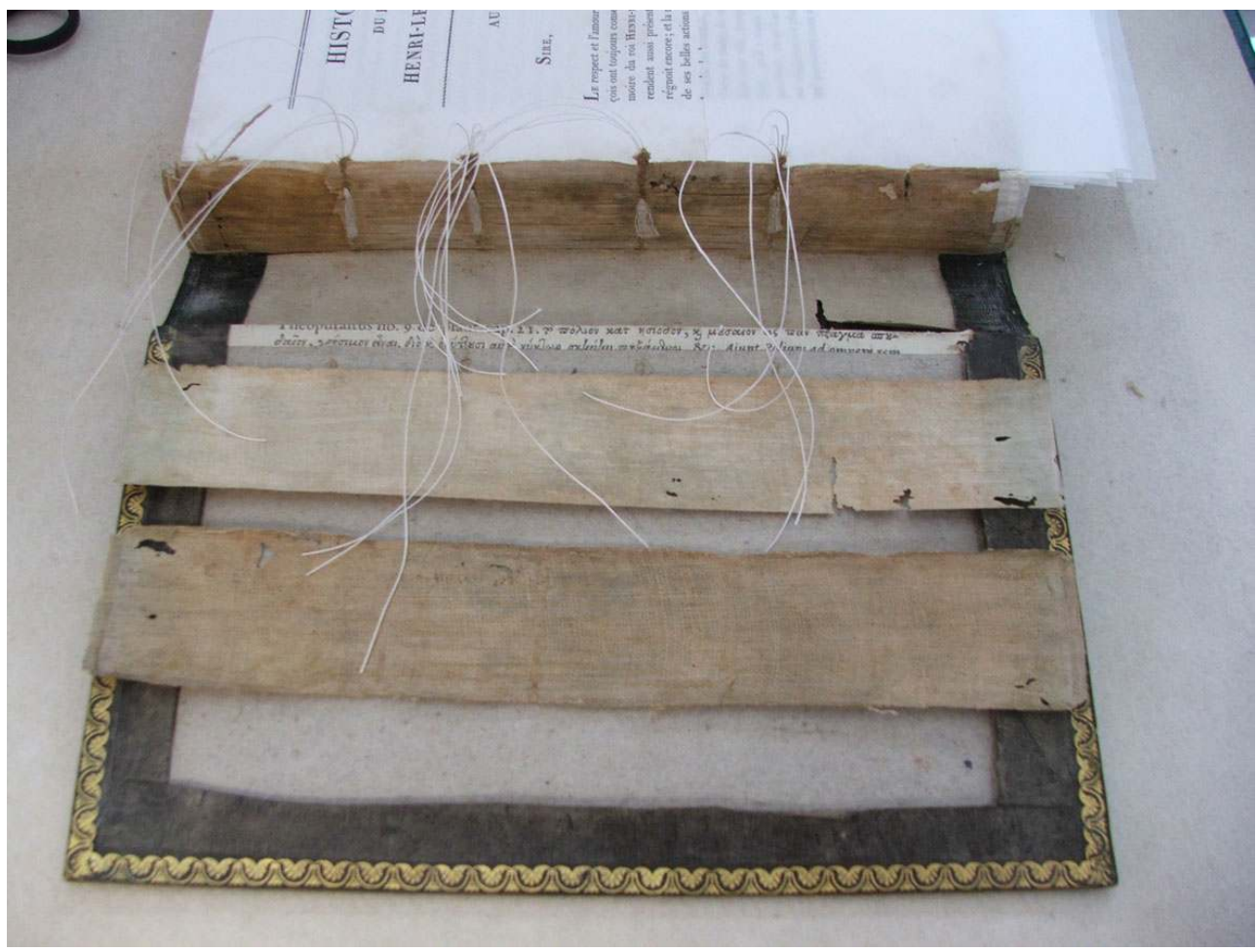

La passure avec des fils de lin

Phot. Laforest, Éric, 2006. (c) Archives nationales 
Figure 28

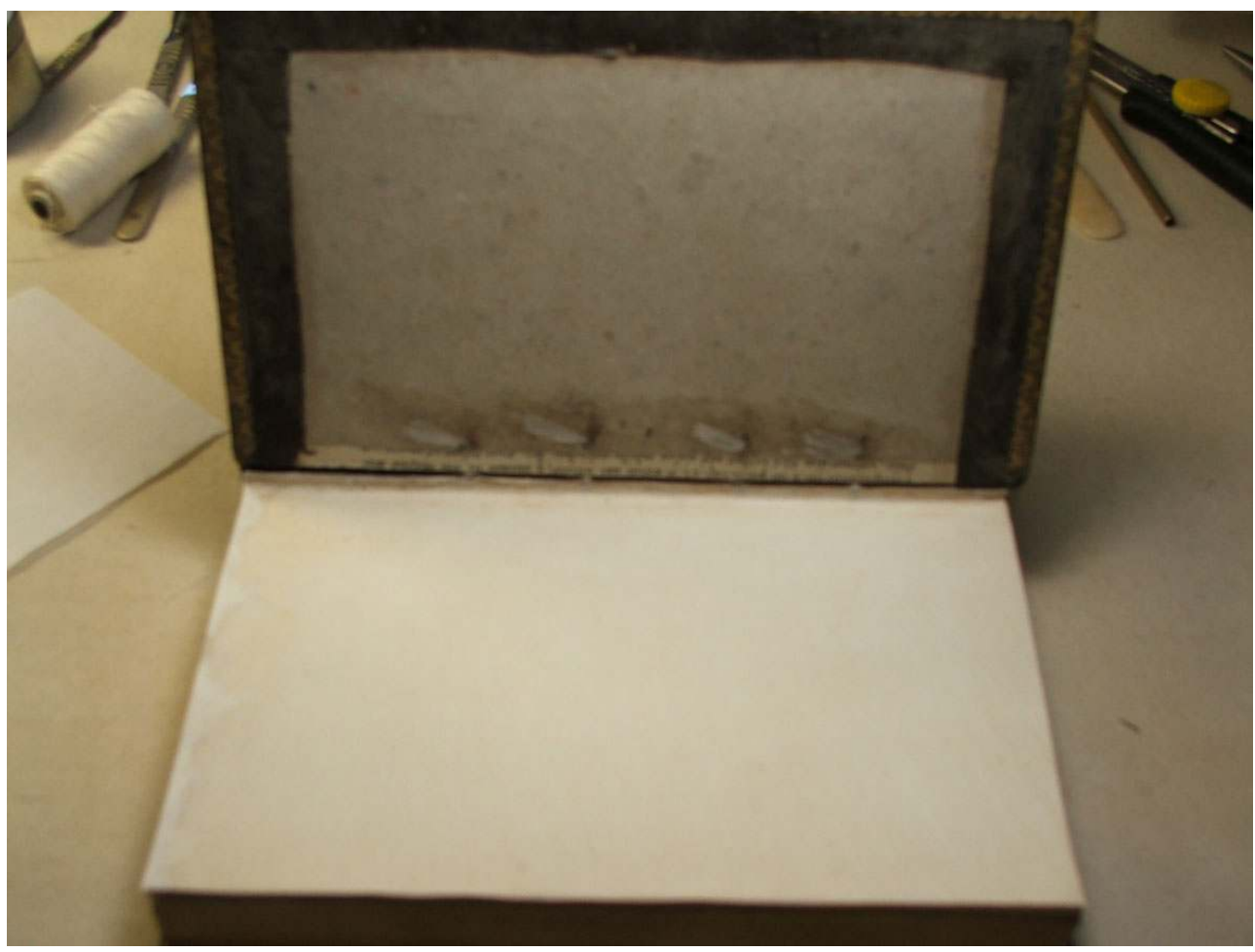

La passure avec des fils de lin

Phot. Laforest, Éric, 2006. ( ) Archives nationales 


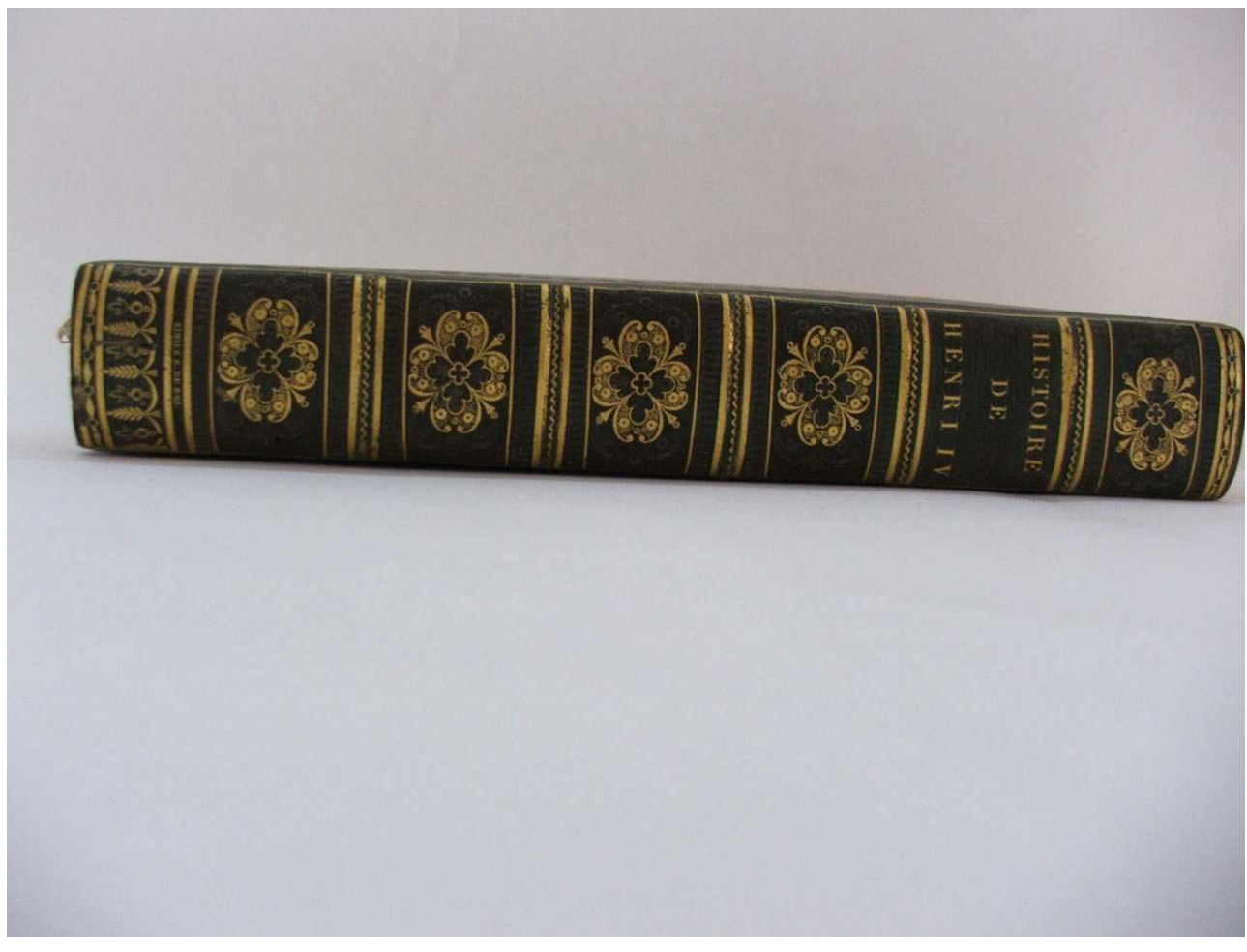

Le dos après réintégration au papier japonais

Phot. Laforest, Éric, 2006. (c) Archives nationales

\section{La conservation}

43 La restauration de ces objets est terminée. Pour les conserver, les ateliers des Archives nationales ont fabriqué sur mesure des conditionnements dont les matériaux répondent aux normes de longue conservation. Ces boîtes trouvent leur place avec les autres objets dans l'Armoire de fer des Archives nationales.

44 Un souci tout particulier a été apporté au choix des couleurs et des textures du conditionnement (cuir vert à grain long). L'atelier de dorure a apporté la touche finale à ce travail de longue haleine en réalisant un décor sur la façade de la boîte qui respecte la scénographie intérieure et l'empreinte stylistique des autres coffres de l'Armoire de fer'?

\section{ANNEXES}




\section{Glossaire}

Apprêter

Coller au dos du volume, un rectangle de toile, de papier ou de parchemin placé en une ou plusieurs couches pour renforcer le dos lors du travail d'apprêture du corps d'ouvrage.

Attache

Lien désignant le type de fixation du corps d'ouvrage aux ais ou aux cartons. Les attaches peuvent être en fil, en cuir, en parchemin, en ficelle, en ruban.

Azurage

Opération consistant à traiter certaines matières (papier, textiles, linge à blanchir, etc.) par des colorants bleus pour supprimer une teinte grise ou jaunâtre et donner plus d'éclat au blanc.

Cahier

Feuille ou partie de feuille de papier qui, après pliure, forme un tout et porte parfois une signature permettant l'assemblage. Un livre est constitué de plusieurs cahiers assemblés et cousus ensemble.

Chagrin

Peau de chèvre présentant un grain accentué et utilisée en reliure.

Charnière

Articulation interne entre le dos et les plats.

Coiffes

Partie du dos en tête et en queue qui recouvre la tranchefile.

Corps d'ouvrage

Ouvrage en cours de travail de reliure, prêt à recevoir sa couvrure.

Couchage

Opération qui consiste à traiter un papier à l'aide de pigments adhésifs, de laque ou de vernis, pour le rendre lustré. Ce procédé rehausse la qualité d'impression.

Couvrure

Pièce de cuir, de parchemin, d'étoffe ou de papier qui recouvre le dos et les plats en plein ou partiellement (demi-reliure). Dans le travail de reliure, la pose de couvrure suit la réalisation du corps d'ouvrage et précède la finissure.

Cuvette

Lors du procédé d'impression, la forme de la plaque gravée laisse sur le papier une empreinte qu'on appelle cuvette.

Dorure

Décoration et titrage de la reliure d'un livre par l'application à chaud, à l'aide de fers gravés en bronze, d'une fine pellicule dorée. Par extension, tous les procédés de décoration d'un livre.

Endossure

Opération qui consiste à pincer le dos pour coucher les cahiers et arrondir le dos, de manière à former les mors du livre, pour y loger les plats. 
Entre-nerfs

Espace compris entre deux nerfs ou faux nerfs sur le dos d'un livre relié.

Faux nerfs

Petites bandelettes de cuir, de carton, de ficelle collées au dos sous la couvrure pour imiter les nerfs sur le dos.

Feuillet

Ensemble de deux pages, recto et verso, d'un livre ou d'un cahier. Isolé, c'est un feuillet volant.

Filets

En dorure, outils en bronze de différentes largeurs et longueurs utilisés pour réaliser les filets qui peuvent être simples ou multiples, dorés ou non.

Fond-tendu

Technique de restauration qui requière l'emploi d'un support rigide (plexiglas, ais, écran sérigraphique) et qui permet les opérations de doublage avec mise en tension.

Format

Dimension d'un livre, souvent désignée par les termes : in-folio, in-quarto, in-octavo, etc., Ces termes font référence au nombre de fois où la feuille a été pliée.

Frontispice

Gravure placée en regard du titre.

Gardes

Feuillets blancs ou de couleurs variées qui commencent ou qui terminent un livre. Ce sont le plus souvent des papiers marbrés. Dans les reliures de luxe, le papier peut être remplacé par des gardes de soie, de velours ou de maroquin.

Gaufrage

Le gaufrage consiste à créer un relief sur une matière.

Gélatine

Substance servant d'agent de collage, de manière à rendre le papier hydrophobe. La gélatine est une substance protéique obtenue par hydrolyse à chaud du collagène présent dans différents tissus animaux. Elle forme une solution colloïdale dans l'eau.

Glaçage

Opération qui consiste à donner (à un objet, à une matière) un aspect lisse et brillant, généralement à l'aide d'un produit spécial. Glaçage d'une feuille, d'un livre, de photographies. Le glaçage facilite l'impression en enlevant au papier son grain et toutes ses rugosités.

Gouttière

Tranche d'un livre opposée au dos et généralement concave.

Grain long

Cuir avec un grainage artificiel formant une succession de traits parallèles.

Grecquer

Consiste à pratiquer sur le fond des cahiers assemblés, mais non cousus, des entailles transversales, dans lesquelles seront logés les supports de couture. Ce terme fait 
référence aux reliures à la grecque, type de reliure en vogue au XVI ${ }^{\mathrm{e}}$ siècle à la cour de Henri II et inspirées des reliures byzantines.

Humidité relative

Le taux d'humidité relative est le rapport entre la quantité de vapeur d'eau qu'un volume d'air donné contient à une température donnée, et la quantité maximale que ce même volume peut contenir à la même température. Cette relation est exprimée en pourcentage.

Karibari

Cadre en bois léger sur lequel est fixée une succession de couches de papier japonais de différents grammages et dans différents sens, servant au doublage et à la mise à plat d'un document sous tension.

Marbrer

Consiste à jeter sur un bain préparé, différentes couleurs que l'on peut mêler pour former des dessins selon le principe de répulsion de l'eau par les matières grasses; et que l'on reportera sur une feuille de papier, sur les tranches d'un livre.

Mors

Épaisseurs longitudinales, formées par l'endossure, dans lesquelles se logent les plats.

Nerfs (ou Nervures)

Les nerfs sont les lanières, les ficelles sur lesquelles on fait passer la couture et qui relient les cahiers ensemble. À l'origine, ils étaient confectionnés à base de peaux, puis on a utilisé du fil de chanvre, de lin, etc.

Parchemin

Peau de veau, de mouton ou de chèvre, préparée spécialement pour recevoir l'écriture. Au Moyen Âge, le parchemin était utilisé pour calligraphier les manuscrits. Plus tard, on l'a utilisé pour relier les livres.

Passure

Action de fixer les cartons sur le volume à relier, en passant les ficelles ou les rubans dans les trous pratiqués à cet effet.

Plat

Éléments en carton ou en bois formant la couverture d'un livre relié et sur lesquels est appliquée la matière de recouvrement : cuir, toile, papier marbré, etc.

Queue

Tranche inférieure d'un livre.

Signet

Petit ruban attaché au dos d'un livre et servant à marquer l'endroit du texte où l'on a interrompu sa lecture. Par extension, morceau de papier marquant une page.

Tête

Tranche supérieure d'un livre.

Tirants

Petits bandes de papier japonais fixées au document et qui aident à effectuer une tension pour une mise à plat.

Tranche

Chacun des trois côtés d'un livre. On parlera de tranche de tête (ou supérieure), tranche 
de queue (ou inférieure), et de gouttière (ou tranche latérale). Elles peuvent être rognées, marbrées, ébarbées, dorées, ciselées, cirées, etc.

Tranchefile

Broderie en fils de soie de couleur placée en tête et en queue du corps d'ouvrage.

\section{NOTES DE FIN}

1. Cette notion est tirée du titre de l'ouvrage de Szirmai, J. The archeology of mediaval boobinding. L'auteur choisit ici un des sens du terme "archéologie " à savoir l'étude des civilisations anciennes réalisée à partir des vestiges matériels d'une activité exercée par les hommes, ou à partir des éléments de leur contexte.

2. Selon les résolutions adoptées par les membres de l'ICOM-CC à l'occasion de la XVe conférence triennale à New Delhi en septembre 2008, http://www.icom-cc.org/.

3. En restauration de livre, c'est Christopher Clarkson qui définit en 1999 l'intervention minimale comme «une interférence minimale» avec les éléments matériels constitutifs du livre. Il argumente en faveur d'une stabilisation mécanique ou chimique des livres qui implique généralement l'adjonction d'une faible quantité de matériaux nouveaux ou étrangers au document original. Clarkson, Christopher, 1999, p. 90.

4. Le traitement de restauration des boîtes en plomb, en bois et en étain a été confié à l'atelier spécialisé Aïnu.

5. Radiographie en trois dimensions.

6. Restauration menée par Laurent Martin Saint-Clair et Isabelle Blivet, techniciens d'art restaurateurs de l'atelier des Archives nationales.

7. Restauration menée par Patricia Coste, technicien d'art restaurateur de l'atelier des Archives nationales.

8. Restauration menée par Éric Laforest, technicien d'art restaurateur de l'atelier de restauration de documents graphiques, de reliures et de dorures des Archives nationales.

9. Dorures réalisées par Anne-Marie Abrard et Christian Jeanjean, techniciens d'art doreurs de l'atelier de restauration des Archives nationales.

\section{RÉSUMÉS}

La rénovation en novembre 2004 de la statue équestre de Henri IV située sur le Pont-Neuf à Paris a permis d'en retirer sept boîtes constituées de bois de cèdre, de buis et de plomb ou d'étain qui renfermaient divers documents. Près de deux siècles séparent la pose des boîtes dans la statue des découvertes réalisées en 2004. Ce long séjour dans des conditions inadaptées n'a pas été sans conséquence pour la conservation des documents. Le Département de la conservation des Archives nationales a décidé de les restaurer et de les conserver comme des objets archéologiques empreints d'une histoire peu commune.

The restoration in November 2004 of the equestrian statue of Henri IV located on the Pont-Neuf in Paris enabled the withdrawal of seven boxes of cedar wood, boxwood and lead or tin which contained various documents. Nearly two centuries passed between the placement of the boxes in the statue and the discoveries made in 2004. This long period in unsuitable conditions had its consequences on the conservation of the documents. The Department of conservation of the National Archives decided to restore them and preserve them as archaeological artifacts imprinted with an unusual history. 
INDEX

Mots-clés : restauration, conservation, parchemin, cuir, papier, dégradation, technique

\section{AUTEURS}

\section{NELLY CAULIEZ}

Chef de travaux d'art, responsable de l'atelier de restauration de documents graphiques, de reliures et de dorures des Archives nationales. nelly.cauliez@culture.gouv.fr

\section{ISABELLE BLIVET}

Technicien d'art, restaurateur-relieur de l'atelier de restauration de documents graphiques, de reliures et de dorures des Archives nationales

\section{MYRIAM KRICHE}

Technicien d'art, restaurateur-relieur de l'atelier de restauration de documents graphiques, de reliures et de dorures des Archives nationales

\section{LAURENT MARTIN-SAINT-CLAIR}

Technicien d'art, restaurateur-relieur de l'atelier de restauration de documents graphiques, de reliures et de dorures des Archives nationales 